\title{
On impulsive nonlocal integro-initial value problems involving multi-order Caputo-type generalized fractional derivatives and generalized fractional integrals
}

\author{
Bashir Ahmad ${ }^{1 *}$ (D, Madeaha Alghanmi ${ }^{1}$, Juan J. Nieto² and Ahmed Alsaedi ${ }^{1}$
}

\section{"Correspondence:}

bashirahmad_qau@yahoo.com ${ }^{1}$ Nonlinear Analysis and Applied Mathematics (NAAM)-Research Group, Department of Mathematics, Faculty of Science, King Abdulaziz University, Jeddah, Saudi Arabia Full list of author information is available at the end of the article

\section{然 Springer}

\begin{abstract}
In this paper, we present sufficient criteria ensuring the existence and uniqueness of solutions for nonlinear impulsive multi-order Caputo-type generalized fractional differential equations supplemented with nonlocal integro-initial value conditions involving generalized fractional integrals. Extremal solutions for the given problem are also discussed. The main tools of our study include Krasnoselskii's fixed point theorem, Banach contraction mapping principle and monotone iterative technique. Examples are constructed for illustrating the obtained results.
\end{abstract}

MSC: 34A08; 34B10; 34B15; 34B37

Keywords: Multi-order fractional derivatives; Impulse; Caputo-type generalized fractional derivative; Fractional integral; Extremal solutions

\section{Introduction}

Impulsive dynamical systems involve some continuous variable dynamic characteristics, together with certain reset maps generating impulsive switching among them. The dynamical behavior of impulsive systems is much more complex than that associated to non-impulsive dynamical systems. Such systems appear in real-time software verification [1], transportation systems [2, 3], automotive control [4, 5], etc. In consequence, the topic of impulsive differential equations has emerged as an important area of investigation as it accounts for several phenomena which are not addressed by the non-impulsive equations.

Arbitrary (non-integer) order differential and integral operators serve as better modeling tools than their corresponding integer-order counterparts, as these operators are capable to retrieve the historical effects of the systems and processes involved in the phenomena. Fractional-order initial and boundary value problems have been investigated by many authors in recent years; for instance, see [6-17].

Factional differential equations with impulse effects also received considerable attention in view of their applications in modeling the physical problems experiencing instantaneous changes. For some recent works on impulsive fractional differential equations, we refer the reader to the papers [18-29] and the references cited therein. In a recent work [26], the authors discussed the existence of extremal solutions for a nonlinear impulsive

(c) The Author(s) 2019. This article is distributed under the terms of the Creative Commons Attribution 4.0 International License (http://creativecommons.org/licenses/by/4.0/), which permits unrestricted use, distribution, and reproduction in any medium, provided you give appropriate credit to the original author(s) and the source, provide a link to the Creative Commons license, and indicate if changes were made. 
differential equations with multi-order fractional derivatives and integral boundary conditions.

In this paper, we introduce a new class of nonlinear nonlocal impulsive multi-order problems involving Caputo-type generalized fractional derivatives and generalized fractional integrals (in the sense of Katugampola). In precise terms, we investigate the following problem:

$$
\begin{cases}{ }_{c}^{\rho} D_{t_{k}^{+}}^{\alpha_{k}} y(t)=f(t, y(t)), & 1<\alpha_{k} \leq 2, k=0,1,2, \ldots, p, t \in J^{\prime}, \\ \triangle y\left(t_{k}\right)=S_{k}\left(y\left(t_{k}\right)\right), \quad \Delta \delta y\left(t_{k}\right)=S_{k}^{*}\left(y\left(t_{k}\right)\right), & k=1,2, \ldots, p, \\ y(0)=\sum_{k=0}^{p} \lambda_{k}{ }^{\rho} I_{t_{k}^{+}}^{\beta_{k}} y\left(\xi_{k}\right)+\eta, \quad \delta y(0)=0, & t_{k}<\xi_{k}<t_{k+1},\end{cases}
$$

where ${ }_{c}^{\rho} D_{t_{k}^{+}}^{\alpha_{k}}$ is the Caputo-type generalized fractional derivative of order $\alpha_{k}, \rho>0,{ }^{\rho} I_{t_{k}^{+}}^{\beta_{k}}$ is the generalized fractional integral of order $\beta_{k}>0, \rho>0$ (defined in the next section), $f \in C(J \times \mathbb{R}, \mathbb{R}), S_{k}, S_{k}^{*} \in C(\mathbb{R}, \mathbb{R}) ; \lambda_{k}, \xi_{k}$ are positive constants; $J=[0, T](T>0), \eta \in \mathbb{R}, 0=$ $t_{0}<t_{1}<\cdots<t_{k}<\cdots<t_{p}<t_{p+1}=T, J^{\prime}=J \backslash\left\{t_{1}, t_{2}, \ldots, t_{m}\right\}, \Delta y\left(t_{k}\right)=y\left(t_{k}^{+}\right)-y\left(t_{k}^{-}\right)$, where $y\left(t_{k}^{+}\right)$ and $y\left(t_{k}^{-}\right)$denote the right and the left limits of $y(t)$ at $t=t_{k}(k=1,2, \ldots, p)$, respectively; $\triangle \delta y\left(t_{k}\right)$ have a similar meaning for $\delta y(t)$, where $\delta=t^{1-\rho} \frac{d}{d t}$.

In Sect. 2, we present the background material related to our work and prove an important lemma which plays a key role in the sequel. Section 3 contains the existence and uniqueness results for problem (1.1). In Sect. 4, we prove a new comparison result and use it to obtain the extremal solutions for problem (1.1).

\section{Preliminaries}

Let us fix $J_{0}=\left[0, t_{1}\right], J_{k}=\left(t_{k}, t_{k+1}\right], k=1,2, \ldots, p$ with $t_{p+1}=T$, and define $P C(J, \mathbb{R})=\{y: J \rightarrow$ $\mathbb{R}: y \in C\left(J_{k}, \mathbb{R}\right), k=0,1, \ldots, p$ and $y\left(t_{k}^{+}\right)$and $y\left(t_{k}^{-}\right)$exist with $\left.y\left(t_{k}^{-}\right)=y\left(t_{k}\right), k=1,2, \ldots, p\right\}$, where $C(J, \mathbb{R})$ denotes the space of all continuous real-valued functions on $J$ and $P C_{\delta}^{1}(J, \mathbb{R})=\left\{y: J \rightarrow \mathbb{R}: \delta y \in P C(J, \mathbb{R}) ; \delta y\left(t_{k}^{+}\right), \delta y\left(t_{k}^{-}\right)\right.$exist and $\delta y$ is left continuous at $t_{k}$ for $\left.k=1,2, \ldots, p, \delta=t^{1-\rho} \frac{d}{d t}\right\}$ with the norm $\|y\|=\sup _{t \in J}\left\{\|y(t)\|_{P C},\|\delta y(t)\|_{P C_{\delta}^{1}}\right\}$. We further recall that $A C^{n}(J, \mathbb{R})=\left\{h: J \rightarrow \mathbb{R}: h, h^{\prime}, \ldots, h^{(n-1)} \in C(J, \mathbb{R})\right.$ and $h^{(n-1)}$ is absolutely continuous $\}$. For $0 \leq \epsilon<1$, we define $C_{\epsilon, \rho}(J, \mathbb{R})=\left\{f: J \rightarrow \mathbb{R}:\left(t^{\rho}-a^{\rho}\right)^{\epsilon} f(t) \in C(J, \mathbb{R})\right\}$ endowed with the norm $\|f\|_{C_{\epsilon, \rho}}=\left\|\left(t^{\rho}-a^{\rho}\right)^{\epsilon} f(t)\right\|_{C}$. Moreover, we define the class of functions $f$ that have absolutely continuous $\delta^{n-1}$-derivative, denoted by $A C_{\delta}^{n}(J, \mathbb{R})$, as follows: $A C_{\delta}^{n}(J, \mathbb{R})=\left\{f: J \rightarrow \mathbb{R}: \delta^{n-1} f \in A C(J, \mathbb{R}), \delta=t^{1-\rho} \frac{d}{d t}\right\}$, which is equipped with the norm $\|f\|_{C_{\delta}^{n}}=\sum_{k=0}^{n-1}\left\|\delta^{k} f\right\|_{C}$. More generally, let $C_{\delta, \epsilon}^{n}(J, \mathbb{R})=\left\{f: J \rightarrow \mathbb{R}: \delta^{n-1} f \in\right.$ $\left.C(J, \mathbb{R}), \delta^{n} f \in C_{\epsilon, \rho}(J, \mathbb{R}), \delta=t^{1-\rho} \frac{d}{d t}\right\}$ be the space of functions endowed with the norm $\|f\|_{C_{\delta, \epsilon}^{n}}=\sum_{k=0}^{n-1}\left\|\delta^{k} f\right\|_{C}+\left\|\delta^{n} f\right\|_{C_{\epsilon, \rho}}$. Here we use the convention $C_{\delta, 0}^{n}=C_{\delta}^{n}$.

For $c \in \mathbb{R}, 1 \leq q \leq \infty$, let $X_{c}^{q}(a, b)$ denote the space of all Lebesgue measurable functions $\phi$ on $(a, b)$ equipped with the norm

$$
\|\phi\|_{X_{c}^{q}}=\left(\int_{a}^{b}\left|x^{c} \phi(x)\right|^{q} \frac{d x}{x}\right)^{1 / q}<\infty .
$$

Definition 2.1 ([30]) The generalized fractional integral of order $\alpha>0$ and $\rho>0$ of $f \in$ $X_{c}^{q}(a, b)$, for $-\infty<a<t<b<\infty$, is defined by

$$
\left({ }^{\rho} I_{a^{+}}^{\alpha} f\right)(t)=\frac{\rho^{1-\alpha}}{\Gamma(\alpha)} \int_{a}^{t} \frac{s^{\rho-1}}{\left(t^{\rho}-s^{\rho}\right)^{1-\alpha}} f(s) d s .
$$


Note that the integral in (2.1) is called the left-sided fractional integral. Similarly, we can define the right-sided fractional integral ${ }^{\rho} I_{b-}^{\alpha} f$ as

$$
\left({ }^{\rho} I_{b^{-}}^{\alpha} f\right)(t)=\frac{\rho^{1-\alpha}}{\Gamma(\alpha)} \int_{t}^{b} \frac{s^{\rho-1}}{\left(s^{\rho}-t^{\rho}\right)^{1-\alpha}} f(s) d s
$$

Definition 2.2 ([31]) The generalized fractional derivatives of $f \in X_{c}^{q}(a, b)$ of order $\alpha \in$ $(n-1, n], n \in \mathbb{N}$, associated with the generalized fractional integrals (2.1) and (2.2), are defined for $0 \leq a<x<b<\infty$ by

$$
\begin{aligned}
\left({ }^{\rho} D_{a^{+}}^{\alpha} f\right)(t) & =\left(t^{1-\rho} \frac{d}{d t}\right)^{n}\left({ }^{\rho} I_{a^{+}}^{n-\alpha} f\right)(t) \\
& =\frac{\rho^{\alpha-n+1}}{\Gamma(n-\alpha)}\left(t^{1-\rho} \frac{d}{d t}\right)^{n} \int_{a}^{t} \frac{s^{\rho-1}}{\left(t^{\rho}-s^{\rho}\right)^{\alpha-n+1}} f(s) d s
\end{aligned}
$$

and

$$
\begin{aligned}
\left({ }^{\rho} D_{b^{-}}^{\alpha} f\right)(t) & =\left(-t^{1-\rho} \frac{d}{d t}\right)^{n}\left({ }^{\rho} I_{b^{-}}^{n-\alpha} f\right)(t) \\
& =\frac{\rho^{\alpha-n+1}}{\Gamma(n-\alpha)}\left(-t^{1-\rho} \frac{d}{d t}\right)^{n} \int_{t}^{b} \frac{s^{\rho-1}}{\left(s^{\rho}-t^{\rho}\right)^{\alpha-n+1}} f(s) d s .
\end{aligned}
$$

Definition 2.3 ([32]) For $\alpha \geq 0$ and $f \in A C_{\delta}^{n}[a, b]$, the Caputo-type generalized fractional derivatives ${ }_{c}^{\rho} D_{a^{+}}^{\alpha}$ and ${ }_{c}^{\rho} D_{b^{-}}^{\alpha}$ are defined in terms of (2.3) and (2.4) as follows:

$$
\begin{aligned}
& { }_{c}^{\rho} D_{a^{+}}^{\alpha} f(x)={ }^{\rho} D_{a^{+}}^{\alpha}\left[f(t)-\sum_{k=0}^{n-1} \frac{\delta^{k} f(a)}{k !}\left(\frac{t^{\rho}-a^{\rho}}{\rho}\right)^{k}\right](x), \quad \delta=x^{1-\rho} \frac{d}{d x}, \\
& { }_{c}^{\rho} D_{b^{-}}^{\alpha} f(x)={ }^{\rho} D_{b^{-}}^{\alpha}\left[f(t)-\sum_{k=0}^{n-1} \frac{(-1)^{k} \delta^{k} f(b)}{k !}\left(\frac{b^{\rho}-t^{\rho}}{\rho}\right)^{k}\right](x), \quad \delta=x^{1-\rho} \frac{d}{d x} .
\end{aligned}
$$

Lemma 2.4 ([32]) Let $\alpha \geq 0, n=[\alpha]+1$, where $[\alpha]$ denotes the integer part of $\alpha$, and $f \in$ $A C_{\delta}^{n}[a, b]$ with $0<a<b<\infty$.

1. If $\alpha \notin \mathbb{N}$, then

$$
\begin{aligned}
& { }_{c}^{\rho} D_{a^{+}}^{\alpha} f(t)=\frac{1}{\Gamma(n-\alpha)} \int_{a}^{t}\left(\frac{t^{\rho}-s^{\rho}}{\rho}\right)^{n-\alpha-1} \frac{\left(\delta^{n} f\right)(s) d s}{s^{1-\rho}}={ }^{\rho} I_{a^{+}}^{n-\alpha}\left(\delta^{n} f\right)(t), \\
& { }_{c}^{\rho} D_{b^{-}}^{\alpha} f(t)=\frac{1}{\Gamma(n-\alpha)} \int_{t}^{b}\left(\frac{s^{\rho}-t^{\rho}}{\rho}\right)^{n-\alpha-1} \frac{(-1)^{n}\left(\delta^{n} f\right)(s) d s}{s^{1-\rho}}={ }^{\rho} I_{b^{-}}^{n-\alpha}\left(\delta^{n} f\right)(t) .
\end{aligned}
$$

2. If $\alpha \in \mathbb{N}$, then

$$
{ }_{c}^{\rho} D_{a^{+}}^{\alpha} f=\delta^{n} f, \quad{ }_{c}^{\rho} D_{b^{-}}^{\alpha} f=(-1)^{n} \delta^{n} f .
$$

Lemma 2.5 ([32]) If $\in A C_{\delta}^{n}[a, b]$ or $C_{\delta}^{n}[a, b]$ and $\alpha \in \mathbb{R}$, then

$$
{ }^{\rho} I_{a^{+} c}^{\alpha}{ }_{c}^{\rho} D_{a^{+}}^{\alpha} f(x)=f(x)-\sum_{k=0}^{n-1} \frac{\left(\delta^{k} f\right)(a)}{k !}\left(\frac{x^{\rho}-a^{\rho}}{\rho}\right)^{k},
$$




$$
{ }^{\rho} I_{b^{-}}^{\alpha}{ }^{\rho} D_{b^{-}}^{\alpha} f(x)=f(x)-\sum_{k=0}^{n-1} \frac{(-1)^{k}\left(\delta^{k} f\right)(a)}{k !}\left(\frac{b^{\rho}-x^{\rho}}{\rho}\right)^{k} .
$$

In particular, for $0<\alpha \leq 1$, we have

$$
\begin{aligned}
& { }^{\rho} I_{a^{+}{ }_{c}{ }^{\rho} D_{a^{f}}^{\alpha} f(x)}^{\alpha}=f(x)-f(a), \\
& { }^{\rho} I_{b^{-}{ }^{\rho}}^{\alpha} D_{b^{-}}^{\alpha} f(x)=f(x)-f(b) .
\end{aligned}
$$

Definition 2.6 A function $y \in P C_{\delta}^{1}(J, \mathbb{R}) \cap A C_{\delta}^{2}\left(J_{k}\right)$ with its Caputo generalized derivative of order $\alpha_{k}, k=0,1, \ldots, p$, is a solution of (1.1) if it satisfies (1.1).

Lemma 2.7 For any $h \in C([0, T], \mathbb{R}), y \in P C_{\delta}^{1}(J, \mathbb{R}) \cap A C_{\delta}^{2}\left(J_{k}\right)$, the constants $S_{k}, S_{k}^{*}(k=$ $1,2, \ldots, p)$ and

$$
\Omega=1-\sum_{k=0}^{p} \frac{\lambda_{k}\left(\xi_{k}^{\rho}-t_{k}^{\rho}\right)^{\beta_{k}}}{\rho^{\beta_{k}} \Gamma\left(\beta_{k}+1\right)} \neq 0
$$

the integral representation of the solution for the following impulsive nonlocal integroinitial value problem

$$
\left\{\begin{array}{l}
{ }_{c}^{\rho} D_{t_{k}^{+}}^{\alpha_{k}} y(t)=h(t), \quad 0<\alpha_{k} \leq 2, k=0,1,2, \ldots, p, t \in J^{\prime}, \\
\triangle y\left(t_{k}\right)=S_{k}, \quad \Delta \delta y\left(t_{k}\right)=S_{k}^{*}, \quad k=1,2, \ldots, p, \\
y(0)=\sum_{k=0}^{p} \lambda_{k}{ }^{\rho} I_{t_{k}^{+}}^{\beta_{k}} y\left(\xi_{k}\right)+\eta, \quad \delta y(0)=0, \quad t_{k}<\xi_{k}<t_{k+1},
\end{array}\right.
$$

is given by

$$
y(t)=\left\{\begin{array}{l}
{ }^{\rho} I_{0^{+}}^{\alpha_{0}} h(t)+A, \quad t \in J_{0}, \\
{ }^{\rho} I_{t_{k}^{+}}^{\alpha_{k}} h(t)+\sum_{i=1}^{k}\left[{ }^{\rho} I_{t_{i-1}^{+}}^{\alpha_{i-1}} h\left(t_{i}\right)+S_{i}\right]+\sum_{i=1}^{k-1}\left(\frac{t_{k}^{\rho}-t_{i}^{\rho}}{\rho}\right)\left[{ }^{\rho} I_{t_{i-1}^{+}}^{\alpha_{i-1}-1} h\left(t_{i}\right)+S_{i}^{*}\right] \\
\left.\quad+\sum_{i=1}^{k} \frac{t^{\rho}-t_{k}^{\rho}}{\rho}\right)\left[{ }^{\rho} I_{t_{i-1}^{+}}^{\alpha_{i-1}-1} h\left(t_{i}\right)+S_{i}^{*}\right]+A, \\
\quad t \in J_{k}, k=1,2, \ldots, p,
\end{array}\right.
$$

where

$$
\begin{aligned}
A= & \frac{1}{\Omega}\left\{\sum_{k=0}^{p} \lambda_{k}{ }^{\rho} I_{t_{k}^{+}}^{\alpha_{k}+\beta_{k}} h\left(\xi_{k}\right)+\sum_{k=1}^{p} \sum_{i=1}^{k} \frac{\lambda_{k}\left(\xi_{k}^{\rho}-t_{k}^{\rho}\right)^{\beta_{k}}}{\rho^{\beta_{k}} \Gamma\left(\beta_{k}+1\right)}\left[{ }^{\rho} I_{t_{i-1}^{+}}^{\alpha_{i-1}} h\left(t_{i}\right)+S_{i}\right]\right. \\
& +\sum_{k=2}^{p} \sum_{i=1}^{k-1} \frac{\lambda_{k}\left(\xi_{k}^{\rho}-t_{k}^{\rho}\right)^{\beta_{k}}\left(t_{k}^{\rho}-t_{i}^{\rho}\right)}{\rho^{\beta_{k}+1} \Gamma\left(\beta_{k}+1\right)}\left[{ }^{\rho} I_{t_{i-1}^{+}}^{\alpha_{i-1}-1} h\left(t_{i}\right)+S_{i}^{*}\right] \\
& \left.+\sum_{k=1}^{p} \sum_{i=1}^{k} \frac{\lambda_{k}\left(\xi_{k}^{\rho}-t_{k}^{\rho}\right)^{\beta_{k}+1}}{\rho^{\beta_{k}+1} \Gamma\left(\beta_{k}+2\right)}\left[{ }^{\rho} I_{t_{i-1}^{+}}^{\alpha_{i-1}-1} h\left(t_{i}\right)+S_{i}^{*}\right]+\eta\right\} .
\end{aligned}
$$

Proof Applying the operator ${ }^{\rho} I_{t_{k}^{+}}^{\alpha_{k}}$ to the fractional differential equation in (2.11) and using Lemma 2.5, we obtain

$$
y(t)={ }^{\rho} I_{t_{k}^{+}}^{\alpha_{k}} h(t)+c_{1, k}+c_{2, k}\left(\frac{t^{\rho}-t_{k}^{\rho}}{\rho}\right)
$$




$$
=\frac{\rho^{1-\alpha_{k}}}{\Gamma\left(\alpha_{k}\right)} \int_{t_{k}}^{t} s^{\rho-1}\left(t^{\rho}-s^{\rho}\right)^{\alpha_{k}-1} h(s) d s+c_{1, k}+c_{2, k}\left(\frac{t^{\rho}-t_{k}^{\rho}}{\rho}\right), \quad t \in J_{k},
$$

where $c_{1, k}, c_{2, k} \in \mathbb{R}, k=0,1, \ldots, p$. Taking $\delta$-derivative of (2.14), we get

$$
\delta y(t)={ }^{\rho} I_{t_{k}^{+}}^{\alpha_{k}-1} h(t)+c_{2, k}=\frac{\rho^{2-\alpha_{k}}}{\Gamma\left(\alpha_{k}-1\right)} \int_{t_{k}}^{t} s^{\rho-1}\left(t^{\rho}-s^{\rho}\right)^{\alpha_{k}-2} h(s) d s+c_{2, k} .
$$

For $t \in J_{0}$, we have

$$
y(t)={ }^{\rho} I_{0^{+}}^{\alpha_{0}} h(t)+c_{1,0}+c_{2,0} \frac{t^{\rho}}{\rho}
$$

and

$$
\delta y(t)={ }^{\rho} I_{0^{+}}^{\alpha_{0}-1} h(t)+c_{2,0} .
$$

Using the condition $\delta y(0)=0$ in (2.17), we get $c_{2,0}=0$. In consequence, (2.16) and (2.17) take the form

$$
y(t)={ }^{\rho} I_{0^{+}}^{\alpha_{0}} h(t)+c_{1,0}, \quad t \in J_{0},
$$

and

$$
\delta y(t)={ }^{\rho} I_{0^{+}}^{\alpha_{0}-1} h(t), \quad t \in J_{0} .
$$

Next, for $t \in J_{1}$, we have

$$
\begin{aligned}
& y(t)={ }^{\rho} I_{t_{1}^{+}}^{\alpha_{1}} h(t)+c_{1,1}+c_{2,1}\left(\frac{t^{\rho}-t_{1}^{\rho}}{\rho}\right), \\
& \delta y(t)={ }^{\rho} I_{t_{1}^{+}}^{\alpha_{1}-1} h(t)+c_{2,1},
\end{aligned}
$$

which imply that

$$
\begin{aligned}
& y\left(t_{1}^{-}\right)={ }^{\rho} I_{0^{+}}^{\alpha_{0}} h\left(t_{1}\right)+c_{1,0}, \quad y\left(t_{1}^{+}\right)=c_{1,1}, \\
& \delta y\left(t_{1}^{-}\right)={ }^{\rho} I_{0^{+}}^{\alpha_{0}-1} h\left(t_{1}\right), \quad \delta y\left(t_{1}^{+}\right)=c_{2,1} .
\end{aligned}
$$

Using the impulse conditions $\triangle y\left(t_{1}\right)=y\left(t_{1}^{+}\right)-y\left(t_{1}^{-}\right)=S_{1}, \Delta \delta y\left(t_{k}\right)=\delta y\left(t_{1}^{+}\right)-\delta y\left(t_{1}^{-}\right)=S_{1}^{*}$ in (2.22) and (2.23), we find that

$$
c_{1,1}={ }^{\rho} I_{0^{+}}^{\alpha_{0}} h\left(t_{1}\right)+c_{1,0}+S_{1}, \quad c_{2,1}={ }^{\rho} I_{0^{+}}^{\alpha_{0}-1} h\left(t_{1}\right)+S_{1}^{*} .
$$

Substituting the values of $c_{1,1}$ and $c_{2,1}$ in (2.20), we obtain

$$
y(t)={ }^{\rho} I_{t_{1}^{+}}^{\alpha_{1}} h(t)+{ }^{\rho} I_{0^{+}}^{\alpha_{0}} h\left(t_{1}\right)+S_{1}+\left(\frac{t^{\rho}-t_{1}^{\rho}}{\rho}\right)\left[{ }^{\rho} I_{0^{+}}^{\alpha_{0}-1} h\left(t_{1}\right)+S_{1}^{*}\right]+c_{1,0}, \quad t \in J_{1} .
$$


By a similar process, for $t \in J_{k}$, we get

$$
\begin{aligned}
y(t)= & { }^{\rho} I_{t_{k}^{+}}^{\alpha_{k}} h(t)+\sum_{i=1}^{k}\left[{ }^{\rho} I_{t_{i-1}^{+}}^{\alpha_{i-1}} h\left(t_{i}\right)+S_{i}\right]+\sum_{i=1}^{k-1}\left(\frac{t_{k}^{\rho}-t_{i}^{\rho}}{\rho}\right)\left[{ }^{\rho} I_{t_{i-1}^{+}}^{\alpha_{i-1}-1} h\left(t_{i}\right)+S_{i}^{*}\right] \\
& +\sum_{i=1}^{k}\left(\frac{t^{\rho}-t_{k}^{\rho}}{\rho}\right)\left[{ }^{\rho} I_{t_{i-1}^{+}}^{\alpha_{i-1}-1} h\left(t_{i}\right)+S_{i}^{*}\right]+c_{1,0}, \quad k=1,2, \ldots, p .
\end{aligned}
$$

For $t \in J_{k}, k=0,1,2, \ldots, p$, we have

$$
\begin{aligned}
{ }^{\rho} I_{t_{k}^{+}}^{\beta_{k}} y(t)= & { }^{\rho} I_{t_{k}^{+}}^{\alpha_{k}+\beta_{k}} h(t)+\sum_{i=1}^{k} \frac{\left(t^{\rho}-t_{k}^{\rho}\right)^{\beta_{k}}}{\rho^{\beta_{k}} \Gamma\left(\beta_{k}+1\right)}\left[{ }^{\rho} I_{t_{i-1}^{+}}^{\alpha_{i-1}} h\left(t_{i}\right)+S_{i}\right] \\
& +\sum_{i=1}^{k-1} \frac{\left(t^{\rho}-t_{k}^{\rho}\right)^{\beta_{k}}\left(t_{k}^{\rho}-t_{i}^{\rho}\right)}{\rho^{\beta_{k}+1} \Gamma\left(\beta_{k}+1\right)}\left[{ }^{\rho} I_{t_{i-1}^{+}}^{\alpha_{i-1}-1} h\left(t_{i}\right)+S_{i}^{*}\right] \\
& +\sum_{i=1}^{k} \frac{\left(t^{\rho}-t_{k}^{\rho}\right)^{\beta_{k}+1}}{\rho^{\beta_{k}+1} \Gamma\left(\beta_{k}+2\right)}\left[{ }^{\rho} I_{t_{i-1}^{+}}^{\alpha_{i-1}-1} h\left(t_{i}\right)+S_{i}^{*}\right]+c_{1,0} \frac{\left(t^{\rho}-t_{k}^{\rho}\right)^{\beta_{k}}}{\rho^{\beta_{k}} \Gamma\left(\beta_{k}+1\right)} .
\end{aligned}
$$

The condition $y(0)=\sum_{k=0}^{p} \lambda_{k} I_{t_{k}^{+}}^{\beta_{k}} y\left(\xi_{k}\right)+\eta$, together with (2.18) and (2.25), implies that

$$
\begin{aligned}
c_{1,0}= & \left(1-\sum_{k=0}^{p} \frac{\lambda_{k}\left(\xi_{k}^{\rho}-t_{k}^{\rho}\right)^{\beta_{k}}}{\rho^{\beta_{k}} \Gamma\left(\beta_{k}+1\right)}\right)^{-1}\left\{\sum_{k=0}^{p} \lambda_{k}{ }^{\rho} I_{t_{k}^{+}}^{\alpha_{k}+\beta_{k}} h\left(\xi_{k}\right)\right. \\
& +\sum_{k=1}^{p} \sum_{i=1}^{k} \frac{\lambda_{k}\left(\xi_{k}^{\rho}-t_{k}^{\rho}\right)^{\beta_{k}}}{\rho^{\beta_{k}} \Gamma\left(\beta_{k}+1\right)}\left[{ }^{\rho} I_{t_{i-1}^{+}}^{\alpha_{i-1}} h\left(t_{i}\right)+S_{i}\right] \\
& +\sum_{k=2}^{p} \sum_{i=1}^{k-1} \frac{\lambda_{k}\left(\xi_{k}^{\rho}-t_{k}^{\rho}\right)^{\beta_{k}}\left(t_{k}^{\rho}-t_{i}^{\rho}\right)}{\rho^{\beta_{k}+1} \Gamma\left(\beta_{k}+1\right)}\left[{ }^{\rho} I_{t_{i-1}^{+}}^{\alpha_{i-1}-1} h\left(t_{i}\right)+S_{i}^{*}\right] \\
& \left.+\sum_{k=1}^{p} \sum_{i=1}^{k} \frac{\lambda_{k}\left(\xi_{k}^{\rho}-t_{k}^{\rho}\right)^{\beta_{k}+1}}{\rho^{\beta_{k}+1} \Gamma\left(\beta_{k}+2\right)}\left[{ }^{\rho} I_{t_{i-1}^{+}}^{\alpha_{i-1}-1} h\left(t_{i}\right)+S_{i}^{*}\right]+\eta\right\}
\end{aligned}
$$

which, on inserting in (2.18) and (2.24), yields the solution (2.12). The converse follows by direct computation. This completes the proof.

\section{Existence and uniqueness results}

In this section, we present the existence and uniqueness results for problem (1.1). Let $\mathcal{G}=P C_{\delta}^{1}(J, \mathbb{R}) \cap A C_{\delta}^{2}\left(J_{k}\right)$. By Lemma 2.7, we transform problem (1.1) into a fixed point problem by defining an operator $F: \mathcal{G} \rightarrow \mathcal{G}$ as

$$
(F y)(t)=\left\{\begin{array}{l}
{ }^{\rho} I_{0^{+}}^{\alpha_{0}} f(t, y(t))+A, \quad t \in J_{0}, \\
{ }^{\rho} I_{t_{k}^{+}}^{\alpha_{k}} f(t, y(t))+\sum_{i=1}^{k}\left[{ }^{\rho} I_{t_{i-1}^{+}}^{\alpha_{i-1}} f\left(t_{i}, y\left(t_{i}\right)\right)+S_{i}\left(y\left(t_{i}\right)\right)\right] \\
\quad+\sum_{i=1}^{k-1}\left(\frac{t_{k}^{\rho}-t_{i}^{\rho}}{\rho}\right)\left[{ }^{\rho} I_{t_{i-1}^{+}}^{\alpha_{i-1}-1} f\left(t_{i}, y\left(t_{i}\right)\right)+S_{i}^{*}\left(y\left(t_{i}\right)\right)\right] \\
\quad+\sum_{i=1}^{k}\left(\frac{t^{\rho}-t_{k}^{\rho}}{\rho}\right)\left[{ }^{\rho} I_{t_{i-1}^{+}}^{\alpha_{i-1}-1} f\left(t_{i}, y\left(t_{i}\right)\right)+S_{i}^{*}\left(y\left(t_{i}\right)\right)\right]+A, \\
\quad t \in J_{k}, k=1,2, \ldots, p,
\end{array}\right.
$$

where $A$ is defined by (2.13). 
For convenience, for $p \geq 1$, we set

$$
\begin{aligned}
& \Lambda_{1}=(1+p) \frac{\max _{0 \leq i \leq p} T^{\alpha_{i} \rho}}{\min _{0 \leq i \leq p}\left\{\rho^{\alpha_{i}} \Gamma\left(\alpha_{i}+1\right)\right\}}+(2 p-1) \frac{\max _{0 \leq i \leq p} T^{\alpha_{i} \rho}}{\min _{0 \leq i \leq p}\left\{\rho^{\alpha_{i}} \Gamma\left(\alpha_{i}\right)\right\}}, \\
& \Lambda_{2}=\frac{1}{|\Omega|}\left\{\sum_{k=0}^{p} \frac{\lambda_{k}\left(\xi_{k}^{\rho}-t_{k}^{\rho}\right)^{\alpha_{k}+\beta_{k}}}{\rho^{\alpha_{k}+\beta_{k}} \Gamma\left(\alpha_{k}+\beta_{k}+1\right)}\right. \\
& +\sum_{k=1}^{p} \sum_{i=1}^{k} \frac{\lambda_{k}\left(\xi_{k}^{\rho}-t_{k}^{\rho}\right)^{\beta_{k}}\left(t_{i}^{\rho}-t_{i-1}^{\rho}\right)^{\alpha_{i-1}}}{\rho^{\beta_{k}+\alpha_{i-1}} \Gamma\left(\beta_{k}+1\right) \Gamma\left(\alpha_{i-1}+1\right)} \\
& +\sum_{k=2}^{p} \sum_{i=1}^{k-1} \frac{\lambda_{k}\left(\xi_{k}^{\rho}-t_{k}^{\rho}\right)^{\beta_{k}}\left(t_{k}^{\rho}-t_{i}^{\rho}\right)\left(t_{i}^{\rho}-t_{i-1}^{\rho}\right)^{\alpha_{i-1}-1}}{\rho^{\beta_{k}+\alpha_{i-1}} \Gamma\left(\beta_{k}+1\right) \Gamma\left(\alpha_{i-1}\right)} \\
& \left.+\sum_{k=1}^{p} \sum_{i=1}^{k} \frac{\lambda_{k}\left(\xi_{k}^{\rho}-t_{k}^{\rho}\right)^{\beta_{k}+1}\left(t_{i}^{\rho}-t_{i-1}^{\rho}\right)^{\alpha_{i-1}-1}}{\rho^{\beta_{k}+\alpha_{i-1}} \Gamma\left(\beta_{k}+2\right) \Gamma\left(\alpha_{i-1}\right)}\right\} \\
& \Lambda_{3}=p+\frac{1}{|\Omega|} \sum_{k=1}^{p} k \frac{\lambda_{k}\left(\xi_{k}^{\rho}-t_{k}^{\rho}\right)^{\beta_{k}}}{\rho^{\beta_{k}} \Gamma\left(\beta_{k}+1\right)}
\end{aligned}
$$

and

$$
\begin{aligned}
\Lambda_{4}= & (2 p-1) \frac{T^{\rho}}{\rho}+\frac{1}{|\Omega|}\left\{\sum_{k=2}^{p} \sum_{i=1}^{k-1} \frac{\lambda_{k}\left(\xi_{k}^{\rho}-t_{k}^{\rho}\right)^{\beta_{k}}\left(t_{k}^{\rho}-t_{i}^{\rho}\right)}{\rho^{\beta_{k}+1} \Gamma\left(\beta_{k}+1\right)}\right. \\
& \left.+\sum_{k=1}^{p} k \frac{\lambda_{k}\left(\xi_{k}^{\rho}-t_{k}^{\rho}\right)^{\beta_{k}+1}}{\rho^{\beta_{k}+1} \Gamma\left(\beta_{k}+2\right)}\right\} .
\end{aligned}
$$

Our first existence result for problem (1.1) relies on Krasnoselskii's fixed point theorem [33], which is stated below.

Lemma 3.1 (Krasnoselskii's fixed point theorem) Suppose $\mathcal{S}$ is a closed convex and nonempty subset of a Banach space X. Let $A, B$ be the operators such that (i) $A x+B y \in \mathcal{S}$ whenever $x, y \in \mathcal{S}$; (ii) $A$ is compact and continuous; and (iii) $B$ is a contraction mapping. Then there exists $w \in \mathcal{S}$ such that $w=A w+B w$.

Theorem 3.2 Let $f:[0, T] \times \mathbb{R} \rightarrow \mathbb{R}$ be a continuous function, and $S_{k}, S_{k}^{*} \in C(\mathbb{R}, \mathbb{R})$. Assume there exist positive constants $L_{2}, L_{3}, M_{2}, M_{3}$ such that the following conditions hold:

$\left(H_{1}\right)\left|S_{k}(x)-S_{k}(y)\right| \leq L_{2}|x-y|,\left|S_{k}^{*}(x)-S_{k}^{*}(y)\right| \leq L_{3}|x-y|$ with $\left\|S_{k}(x)\right\| \leq M_{2},\left\|S_{k}^{*}(x)\right\| \leq$ $M_{3}, \forall x, y \in \mathbb{R}, k=1,2, \ldots, p ;$

$\left(H_{2}\right)|f(t, y)| \leq \phi(t), \forall(t, y) \in[0, T] \times \mathbb{R}$, and $\phi \in C\left([0, T], \mathbb{R}^{+}\right)$.

Then problem (1.1) has at least one solution on J, provided that

$$
L_{2} \Lambda_{3}+L_{3} \Lambda_{4}<1
$$


Proof Consider $B_{r}=\{y \in \mathcal{G}:\|y\| \leq r\}$ with $r>\|\phi\|\left(\Lambda_{1}+\Lambda_{2}\right)+M_{2} \Lambda_{3}+M_{3} \Lambda_{4}+\frac{|\eta|}{|\Omega|},\|\phi\|=$ $\sup _{t \in[0, T]}|\phi(t)|$ and define operators $\mathcal{P}$ and $\mathcal{Q}$ on $B_{r}$ as follows:

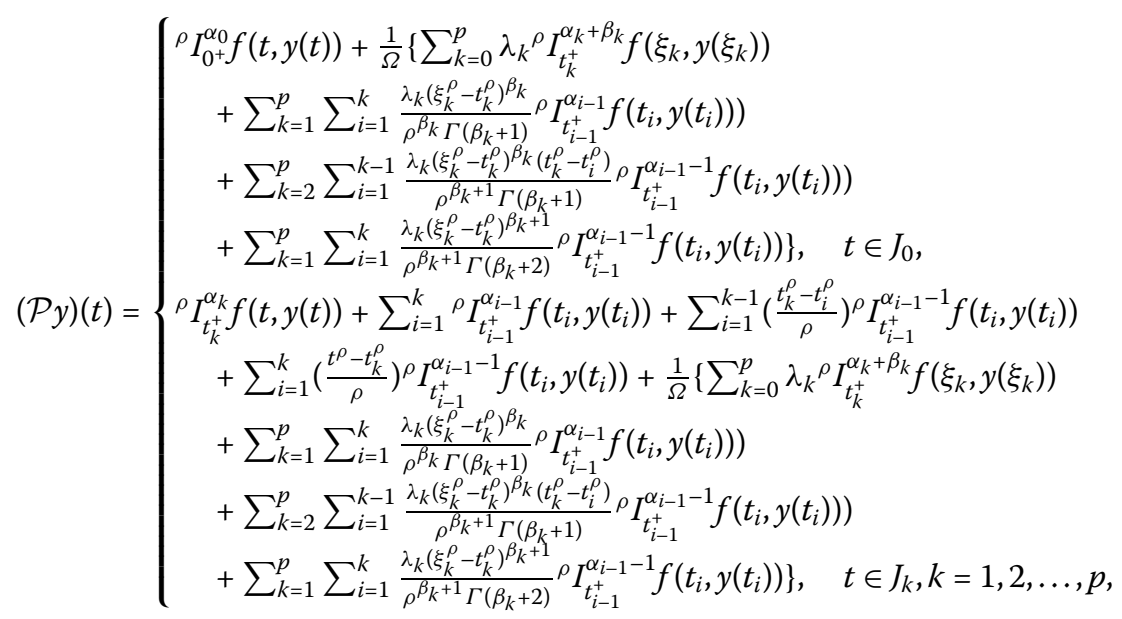

and

$$
(\mathcal{Q} y)(t)=\left\{\begin{aligned}
\frac{1}{\Omega}\left\{\sum_{k=1}^{p} \sum_{i=1}^{k} \frac{\lambda_{k}\left(\xi_{k}^{\rho}-t_{k}^{\rho}\right)^{\beta_{k}}}{\rho^{\beta_{k}} \Gamma\left(\beta_{k}+1\right)} S_{i}\left(y\left(t_{i}\right)\right)+\sum_{k=2}^{p} \sum_{i=1}^{k-1} \frac{\lambda_{k}\left(\xi_{k}^{\rho}-t_{k}^{\rho} \beta_{k}\left(t_{k}^{\rho}-t_{i}^{\rho}\right)\right.}{\rho^{\beta_{k}+1} \Gamma\left(\beta_{k}+1\right)} S_{i}^{*}\left(y\left(t_{i}\right)\right)\right. \\
\left.\quad+\sum_{k=1}^{p} \sum_{i=1}^{k} \frac{\lambda_{k}\left(\xi_{k}-t_{k}^{\rho} \beta_{k}+1\right.}{\rho^{\beta_{k}+1} \Gamma\left(\beta_{k}+2\right)} S_{i}^{*}\left(y\left(t_{i}\right)\right)+\eta\right\}, \quad t \in J_{0}, \\
\sum_{i=1}^{k} S_{i}\left(y\left(t_{i}\right)\right)+\sum_{i=1}^{k-1}\left(\frac{t_{k}^{\rho}-t_{i}^{\rho}}{\rho}\right) S_{i}^{*}\left(y\left(t_{i}\right)\right)+\sum_{i=1}^{k}\left(\frac{t^{\rho}-t_{k}^{\rho}}{\rho}\right) S_{i}^{*}\left(y\left(t_{i}\right)\right) \\
\quad+\frac{1}{\Omega}\left\{\sum_{k=1}^{p} \sum_{i=1}^{k} \frac{\lambda_{k}\left(\xi_{k}^{\rho}-t_{k}^{\rho}\right)^{\beta_{k}}}{\rho^{\beta} \beta_{k}\left(\beta_{k}+1\right)} S_{i}\left(y\left(t_{i}\right)\right)+\sum_{k=2}^{p} \sum_{i=1}^{k-1} \frac{\lambda_{k}\left(\xi_{k}^{\rho}-t_{k}^{\rho} \beta_{k}\left(t_{k}^{\rho}-t_{i}^{\rho}\right)\right.}{\rho^{\beta} \beta_{k}^{+1} \Gamma\left(\beta_{k}+1\right)} S_{i}^{*}\left(y\left(t_{i}\right)\right)\right. \\
\left.\quad+\sum_{k=1}^{p} \sum_{i=1}^{k} \frac{\lambda_{k}\left(\xi_{k}^{\rho}-t_{k}^{\rho} \beta_{k}+1\right.}{\rho^{\beta} k^{+1} \Gamma\left(\beta_{k}+2\right)} S_{i}^{*}\left(y\left(t_{i}\right)\right)+\eta\right\}, \quad t \in J_{k}, k=1,2, \ldots, p .
\end{aligned}\right.
$$

Observe that $\mathcal{P}+\mathcal{Q}=F$, where the operator $F: \mathcal{G} \rightarrow \mathcal{G}$ is defined by (3.1). For $x, y \in B_{r}$ and $t \in J_{0}$, we have

$$
\begin{aligned}
\| \mathcal{P} x & +\mathcal{Q} y \| \\
\leq & \sup _{t \in J}\left\{{ }^{\rho} I_{0^{+}}^{\alpha_{0}}|f(t, x(t))|+\frac{1}{|\Omega|}\left\{\sum_{k=0}^{p} \lambda_{k}{ }^{\rho} I_{t_{k}^{+}}^{\alpha_{k}+\beta_{k}}\left|f\left(\xi_{k}, x\left(\xi_{k}\right)\right)\right|\right.\right. \\
& +\sum_{k=1}^{p} \sum_{i=1}^{k} \frac{\lambda_{k}\left(\xi_{k}^{\rho}-t_{k}^{\rho}\right)^{\beta_{k}}}{\rho^{\beta_{k}} \Gamma\left(\beta_{k}+1\right)}\left[{ }^{\rho} I_{t_{i-1}^{+}}^{\alpha_{i-1}}\left|f\left(t_{i}, x\left(t_{i}\right)\right)\right|+\left|S_{i}\left(y\left(t_{i}\right)\right)\right|\right] \\
& +\sum_{k=2}^{p} \sum_{i=1}^{k-1} \frac{\lambda_{k}\left(\xi_{k}^{\rho}-t_{k}^{\rho}\right)^{\beta_{k}}\left(t_{k}^{\rho}-t_{i}^{\rho}\right)}{\rho^{\beta_{k}+1} \Gamma\left(\beta_{k}+1\right)}\left[{ }^{\rho} I_{t_{i-1}^{\alpha_{i-1}}}^{\alpha_{i-1}}\left|f\left(t_{i}, x\left(t_{i}\right)\right)\right|+\left|S_{i}^{*}\left(y\left(t_{i}\right)\right)\right|\right] \\
& \left.\left.+\sum_{k=1}^{p} \sum_{i=1}^{k} \frac{\lambda_{k}\left(\xi_{k}^{\rho}-t_{k}^{\rho}\right)^{\beta_{k}+1}}{\rho^{\beta_{k}+1} \Gamma\left(\beta_{k}+2\right)}\left[{ }^{\rho} I_{t_{i-1}}^{\alpha_{i-1}-1}\left|f\left(t_{i}, x\left(t_{i}\right)\right)\right|+\left|S_{i}^{*}\left(y\left(t_{i}\right)\right)\right|\right]+|\eta|\right\}\right\} \\
\leq & \|\phi\|\left(\Lambda_{1}+\Lambda_{2}\right)+M_{2} \Lambda_{3}+M_{3} \Lambda_{4}+\frac{|\eta|}{|\Omega|}<r .
\end{aligned}
$$

Next, for $x, y \in B_{r}$ and $t \in J_{k}, k=1,2, \ldots, p$, we obtain

$$
\|\mathcal{P} x+\mathcal{Q} y\|
$$




$$
\begin{aligned}
& \leq \sup _{t \in J}\left\{{ }^{\rho} I_{t_{k}^{+}}^{\alpha_{k}}|f(t, x(t))|+\sum_{i=1}^{k}\left[{ }^{\rho} I_{t_{i-1}^{+}}^{\alpha_{i-1}}\left|f\left(t_{i}, x\left(t_{i}\right)\right)\right|+\left|S_{i}\left(y\left(t_{i}\right)\right)\right|\right]\right. \\
& +\sum_{i=1}^{k-1}\left(\frac{t_{k}^{\rho}-t_{i}^{\rho}}{\rho}\right)\left[{ }^{\rho}{ }_{t_{i-1}^{+}}^{\alpha_{i-1}-1}\left|f\left(t_{i}, x\left(t_{i}\right)\right)\right|+\left|S_{i}^{*}\left(y\left(t_{i}\right)\right)\right|\right] \\
& +\sum_{i=1}^{k}\left(\frac{t^{\rho}-t_{k}^{\rho}}{\rho}\right)\left[{ }^{\rho} I_{t_{i-1}^{+}}^{\alpha_{i-1}-1}\left|f\left(t_{i}, x\left(t_{i}\right)\right)\right|\right. \\
& \left.+\left|S_{i}^{*}\left(y\left(t_{i}\right)\right)\right|\right]+\frac{1}{|\Omega|}\left\{\sum_{k=0}^{p} \lambda_{k}{ }^{\rho} I_{t_{k}^{+}}^{\alpha_{k}+\beta_{k}}\left|f\left(\xi_{k}, x\left(\xi_{k}\right)\right)\right|\right. \\
& +\sum_{k=1}^{p} \sum_{i=1}^{k} \frac{\lambda_{k}\left(\xi_{k}^{\rho}-t_{k}^{\rho}\right)^{\beta_{k}}}{\rho^{\beta_{k}} \Gamma\left(\beta_{k}+1\right)}\left[{ }^{\rho} I_{t_{i-1}^{+}}^{\alpha_{i-1}}\left|f\left(t_{i}, x\left(t_{i}\right)\right)\right|+\left|S_{i}\left(y\left(t_{i}\right)\right)\right|\right] \\
& +\sum_{k=2}^{p} \sum_{i=1}^{k-1} \frac{\lambda_{k}\left(\xi_{k}^{\rho}-t_{k}^{\rho}\right)^{\beta_{k}}\left(t_{k}^{\rho}-t_{i}^{\rho}\right)}{\rho^{\beta_{k}+1} \Gamma\left(\beta_{k}+1\right)}\left[{ }^{\rho} I_{t_{i-1}^{+}}^{\alpha_{i-1}-1}\left|f\left(t_{i}, x\left(t_{i}\right)\right)\right|+\left|S_{i}^{*}\left(y\left(t_{i}\right)\right)\right|\right] \\
& \left.\left.+\sum_{k=1}^{p} \sum_{i=1}^{k} \frac{\lambda_{k}\left(\xi_{k}^{\rho}-t_{k}^{\rho}\right)^{\beta_{k}+1}}{\rho^{\beta_{k}+1} \Gamma\left(\beta_{k}+2\right)}\left[{ }^{\rho} I_{t_{i-1}^{+}}^{\alpha_{i-1}-1}\left|f\left(t_{i}, x\left(t_{i}\right)\right)\right|+\left|S_{i}^{*}\left(y\left(t_{i}\right)\right)\right|\right]+|\eta|\right\}\right\} \\
& \leq\|\phi\|\left(\Lambda_{1}+\Lambda_{2}\right)+M_{2} \Lambda_{3}+M_{3} \Lambda_{4}+\frac{|\eta|}{|\Omega|}<r .
\end{aligned}
$$

Thus, $\mathcal{P} x+\mathcal{Q} y \in B_{r}$. It follows from the assumptions $\left(H_{1}\right)$ and (3.6) that $\mathcal{Q}$ is a contraction, that is, for $x, y \in B_{r}$ and $t \in J_{0}$, we have

$$
\begin{aligned}
\| \mathcal{Q} y & -\mathcal{Q} z \| \\
\leq & \sup _{t \in J}\left\{\frac { 1 } { | \Omega | } \left\{\sum_{k=1}^{p} \sum_{i=1}^{k} \frac{\lambda_{k}\left(\xi_{k}^{\rho}-t_{k}^{\rho}\right)^{\beta_{k}}}{\rho^{\beta_{k}} \Gamma\left(\beta_{k}+1\right)}\left|S_{i}\left(y\left(t_{i}\right)\right)-S_{i}\left(z\left(t_{i}\right)\right)\right|\right.\right. \\
& +\sum_{k=2}^{p} \sum_{i=1}^{k-1} \frac{\lambda_{k}\left(\xi_{k}^{\rho}-t_{k}^{\rho}\right)^{\beta_{k}}\left(t_{k}^{\rho}-t_{i}^{\rho}\right)}{\rho^{\beta_{k}+1} \Gamma\left(\beta_{k}+1\right)} \\
& \left.\left.\times\left|S_{i}^{*}\left(y\left(t_{i}\right)\right)-S_{i}^{*}\left(z\left(t_{i}\right)\right)\right|+\sum_{k=1}^{p} \sum_{i=1}^{k} \frac{\lambda_{k}\left(\xi_{k}^{\rho}-t_{k}^{\rho}\right)^{\beta_{k}+1}}{\rho^{\beta_{k}+1} \Gamma\left(\beta_{k}+2\right)}\left|S_{i}^{*}\left(y\left(t_{i}\right)\right)-S^{*}\left(z\left(t_{i}\right)\right)\right|\right\}\right\} \\
\leq & L_{2}\|y-z\| \frac{1}{|\Omega|}\left\{\sum_{k=1}^{p} \sum_{i=1}^{k} \frac{\lambda_{k}\left(\xi_{k}^{\rho}-t_{k}^{\rho}\right)^{\beta_{k}}}{\rho^{\beta_{k}} \Gamma\left(\beta_{k}+1\right)}\right\} \\
& +L_{3}\|y-z\| \frac{1}{|\Omega|}\left\{\sum_{k=2}^{p} \sum_{i=1}^{k-1} \frac{\lambda_{k}\left(\xi_{k}^{\rho}-t_{k}^{\rho}\right)^{\beta_{k}}\left(t_{k}^{\rho}-t_{i}^{\rho}\right)}{\rho^{\beta_{k}+1} \Gamma\left(\beta_{k}+1\right)}\right. \\
& \left.+\sum_{k=1}^{p} k \frac{\lambda_{k}\left(\xi_{k}^{\rho}-t_{k}^{\rho}\right)^{\beta_{k}+1}}{\rho^{\beta_{k}+1} \Gamma\left(\beta_{k}+2\right)}\right\} \\
\leq & \left\{L_{2} \Lambda_{3}+L_{3} \Lambda_{4}\right\}\|x-y\| .
\end{aligned}
$$

Similarly, for $x, y \in B_{r}$ and $t \in J_{k}$, one can obtain

$$
\|\mathcal{Q} y-\mathcal{Q} z\|
$$




$$
\begin{aligned}
\leq & \sup _{t \in J}\left\{\sum_{i=1}^{k}\left|S_{i}\left(y\left(t_{i}\right)\right)-S_{i}\left(z\left(t_{i}\right)\right)\right|+\sum_{i=1}^{k-1}\left(\frac{t_{k}^{\rho}-t_{i}^{\rho}}{\rho}\right)\left|S_{i}^{*}\left(y\left(t_{i}\right)\right)-S_{i}^{*}\left(z\left(t_{i}\right)\right)\right|\right. \\
& +\sum_{i=1}^{k}\left(\frac{t^{\rho}-t_{k}^{\rho}}{\rho}\right)\left|S_{i}^{*}\left(y\left(t_{i}\right)\right)-S_{i}^{*}\left(z\left(t_{i}\right)\right)\right| \\
& +\frac{1}{|\Omega|}\left\{\sum_{k=1}^{p} \sum_{i=1}^{k} \frac{\lambda_{k}\left(\xi_{k}^{\rho}-t_{k}^{\rho}\right)^{\beta_{k}}}{\rho^{\beta_{k}} \Gamma\left(\beta_{k}+1\right)}\left|S_{i}\left(y\left(t_{i}\right)\right)-S_{i}\left(z\left(t_{i}\right)\right)\right|\right. \\
& +\sum_{k=2}^{p} \sum_{i=1}^{k-1} \frac{\lambda_{k}\left(\xi_{k}^{\rho}-t_{k}^{\rho}\right)^{\beta_{k}}\left(t_{k}^{\rho}-t_{i}^{\rho}\right)}{\rho^{\beta_{k}+1} \Gamma\left(\beta_{k}+1\right)}\left|S_{i}^{*}\left(y\left(t_{i}\right)\right)-S_{i}^{*}\left(z\left(t_{i}\right)\right)\right| \\
& \left.\left.+\sum_{k=1}^{p} \sum_{i=1}^{k} \frac{\lambda_{k}\left(\xi_{k}^{\rho}-t_{k}^{\rho}\right)^{\beta_{k}+1}}{\rho^{\beta_{k}+1} \Gamma\left(\beta_{k}+2\right)}\left|S_{i}^{*}\left(y\left(t_{i}\right)\right)-S^{*}\left(z\left(t_{i}\right)\right)\right|\right\}\right\} \\
\leq & L_{2}\|y-z\|\left\{p+\frac{1}{|\Omega|}\left\{\sum_{k=1}^{p} \sum_{i=1}^{k} \frac{\lambda_{k}\left(\xi_{k}^{\rho}-t_{k}^{\rho}\right)^{\beta_{k}}}{\rho^{\beta_{k}} \Gamma\left(\beta_{k}+1\right)}\right\}\right\}+L_{3}\|y-z\|\left\{(2 p-1) \frac{T^{\rho}}{\rho}\right. \\
& +\frac{1}{|\Omega|}\left\{\sum_{k=2}^{p} \sum_{i=1}^{k-1} \frac{\lambda_{k}\left(\xi_{k}^{\rho}-t_{k}^{\rho}\right)^{\beta_{k}}\left(t_{k}^{\rho}-t_{i}^{\rho}\right)}{\rho^{\beta_{k}+1} \Gamma\left(\beta_{k}+1\right)}+\sum_{k=1}^{p} k \frac{\lambda_{k}\left(\xi_{k}^{\rho}-t_{k}^{\rho}\right)^{\beta_{k}+1}}{\rho^{\beta_{k}+1} \Gamma\left(\beta_{k}+2\right)}\right\} \\
\leq & \left\{L_{2} \Lambda_{3}+L_{3} \Lambda_{4}\right\}\|x-y\| .
\end{aligned}
$$

Continuity of $f$ implies that operator $\mathcal{P}$ is continuous. Also, $\mathcal{P}$ is uniformly bounded on $B_{r}$ as

$$
\|\mathcal{P} y\| \leq\|\phi\|\left(\Lambda_{1}+\Lambda_{2}\right) .
$$

In order to prove the compactness of operator $\mathcal{P}$, let $\sup _{(t, y) \in J \times B_{r}}|f(t, y)|=\bar{f}<\infty$. Then, for $\tau_{1}, \tau_{2} \in J_{0}$ with $\tau_{1}<\tau_{2}$, we have

$$
\begin{aligned}
\left|(\mathcal{P} y)\left(\tau_{2}\right)-(\mathcal{P} y)\left(\tau_{1}\right)\right| & \\
= & \mid \frac{\rho^{1-\alpha_{0}}}{\Gamma\left(\alpha_{0}\right)}\left[\int_{0}^{\tau_{1}} s^{\rho-1}\left[\left(\tau_{2}^{\rho}-s^{\rho}\right)^{\alpha_{0}-1}-\left(\tau_{1}^{\rho}-s^{\rho}\right)^{\alpha_{0}-1}\right] f(s, y(s)) d s\right. \\
& \left.\quad+\int_{\tau_{1}}^{\tau_{2}} s^{\rho-1}\left(\tau_{2}^{\rho}-s^{\rho}\right)^{\alpha_{0}-1} f(s, y(s)) d s\right] \mid \\
\leq & \frac{\bar{f}}{\rho^{\alpha_{0}} \Gamma\left(\alpha_{0}+1\right)}\left\{2\left(\tau_{2}^{\rho}-\tau_{1}^{\rho}\right)^{\alpha_{0}}+\left|\tau_{2}^{\rho \alpha_{0}}-\tau_{1}^{\rho \alpha_{0}}\right|\right\} .
\end{aligned}
$$

Also, for $\tau_{1}, \tau_{2} \in J_{k}, k=1,2, \ldots, p\left(\tau_{1}<\tau_{2}\right)$, we get

$$
\begin{aligned}
& \left|(\mathcal{P} y)\left(\tau_{2}\right)-(\mathcal{P} y)\left(\tau_{1}\right)\right| \\
& =\mid \frac{\rho^{1-\alpha_{k}}}{\Gamma\left(\alpha_{k}\right)}\left[\int_{t_{k}}^{\tau_{1}} s^{\rho-1}\left[\left(\tau_{2}^{\rho}-s^{\rho}\right)^{\alpha_{k}-1}-\left(\tau_{1}^{\rho}-s^{\rho}\right)^{\alpha_{k}-1}\right] f(s, y(s)) d s\right. \\
& \left.\quad+\int_{\tau_{1}}^{\tau_{2}} s^{\rho-1}\left(\tau_{2}^{\rho}-s^{\rho}\right)^{\alpha_{k}-1} f(s, y(s)) d s\right]
\end{aligned}
$$




$$
\begin{aligned}
& +\sum_{i=1}^{k}\left(\frac{\left(\tau_{2}^{\rho}-t_{k}^{\rho}\right)-\left(\tau_{1}^{\rho}-t_{k}^{\rho}\right)}{\rho}\right)\left(\frac{\rho^{2-\alpha_{i}}}{\Gamma\left(\alpha_{i}-1\right)} \int_{t_{i-1}}^{t_{i}} s^{\rho-1}\left(t_{i}^{\rho}-s^{\rho}\right)^{\alpha_{i}-2} f(s, y(s)) d s\right) \mid \\
\leq & \frac{\bar{f}}{\rho^{\alpha_{k}} \Gamma\left(\alpha_{k}+1\right)}\left\{2\left(\tau_{2}^{\rho}-\tau_{1}^{\rho}\right)^{\alpha_{k}}+\left|\left(\tau_{2}^{\rho}-t_{k}^{\rho}\right)^{\alpha_{k}}-\left(\tau_{1}^{\rho}-t_{k}^{\rho}\right)^{\alpha_{k}}\right|\right\} \\
& +\bar{f} \sum_{i=1}^{k}\left(\frac{\left[\left(\tau_{2}^{\rho}-t_{k}^{\rho}\right)-\left(\tau_{1}^{\rho}-t_{k}^{\rho}\right)\right]\left(t_{i}^{\rho}-t_{i-1}^{\rho}\right)^{\alpha_{i}-1}}{\rho^{\alpha_{i}} \Gamma\left(\alpha_{i}\right)}\right) .
\end{aligned}
$$

From the above inequalities, it follows that $\left|(\mathcal{P} y)\left(\tau_{2}\right)-(\mathcal{P} y)\left(\tau_{1}\right)\right| \rightarrow 0$ as $\tau_{2}-\tau_{1} \rightarrow$ $0, \forall \tau_{1}, \tau_{2} \in J_{k}, k=0,1, \ldots, p$, independent of $y$. Thus, $\mathcal{P}$ is equicontinuous. So $\mathcal{P}$ is relatively compact on $B_{r}$. Hence, by the Arzelá-Ascoli theorem, $\mathcal{P}$ is compact on $B_{r}$. Thus all the assumptions of Lemma 3.1 are satisfied. Hence the conclusion of Lemma 3.1 applies, and so the boundary value problem (1.1) has at least one solution on $J$.

In the following result, we establish the uniqueness of solutions for problem (1.1) with the aid of the contraction mapping principle.

Theorem 3.3 Suppose $f \in C(J \times \mathbb{R}, \mathbb{R})$, assumption $\left(H_{1}\right)$ holds, and the following condition is satisfied:

$\left(H_{3}\right)$ there exists a positive constant $L_{1}$ such that

$$
|f(t, x)-f(t, y)| \leq L_{1}|x-y|, \quad \text { for } t \in J \text { and every } x, y \in \mathbb{R} .
$$

Then there exists a unique solution for problem (1.1) on J if

$$
L_{1}\left(\Lambda_{1}+\Lambda_{2}\right)+L_{2} \Lambda_{3}+L_{3} \Lambda_{4}<1
$$

where $\Lambda_{1}, \Lambda_{2}, \Lambda_{3}$, and $\Lambda_{4}$ are given by (3.2), (3.3), (3.4), and (3.5), respectively.

Proof Setting $\sup _{t \in J}|f(t, 0)|=M_{1}$, we consider the set $B_{r}=\{y \in \mathcal{G}:\|y\| \leq r\}$ with

$$
r>\frac{M_{1}\left(\Lambda_{1}+\Lambda_{2}\right)+M_{2} \Lambda_{3}+M_{3} \Lambda_{4}+\frac{|\eta|}{|\Omega|}}{1-L_{1}\left(\Lambda_{1}+\Lambda_{2}\right)}
$$

and show that $F B_{r} \subset B_{r}$. For $y \in B_{r}$ and $t \in J_{0}$, we have

$$
\begin{aligned}
|(F y)(t)| & =\left|{ }^{\rho} I_{0^{+}}^{\alpha_{0}} f(t, y(t))+A\right| \\
\leq & { }^{\rho} I_{0^{+}}^{\alpha_{0}}[|f(t, y(t))-f(t, 0)|+|f(t, 0)|] \\
& +\frac{1}{|\Omega|}\left\{\sum_{k=0}^{p} \lambda_{k}{ }^{\rho} I_{t_{k}^{+}}^{\alpha_{k}+\beta_{k}}\left[\left|f\left(\xi_{k}, y\left(\xi_{k}\right)\right)-f\left(\xi_{k}, 0\right)\right|+\left|f\left(\xi_{k}, 0\right)\right|\right]\right. \\
& +\sum_{k=1}^{p} \sum_{i=1}^{k} \frac{\lambda_{k}\left(\xi_{k}^{\rho}-t_{k}^{\rho}\right)^{\beta_{k}}}{\rho^{\beta_{k}} \Gamma\left(\beta_{k}+1\right)} \\
& \times\left[{ }^{\rho} I_{t_{i-1}}^{\alpha_{i-1}}\left[\left|f\left(t_{i}, y\left(t_{i}\right)\right)-f\left(t_{i}, 0\right)\right|+\left|f\left(t_{i}, 0\right)\right|\right]+\mid S_{i}\left(y\left(t_{i}\right) \mid\right]\right. \\
& +\sum_{k=2}^{p} \sum_{i=1}^{k-1} \frac{\lambda_{k}\left(\xi_{k}^{\rho}-t_{k}^{\rho}\right)^{\beta_{k}}\left(t_{k}^{\rho}-t_{i}^{\rho}\right)}{\rho^{\beta_{k}+1} \Gamma\left(\beta_{k}+1\right)}
\end{aligned}
$$




$$
\begin{aligned}
& \times\left[{ }_{t_{i-1}^{+}}^{\alpha_{i-1}-1}\left[\left|f\left(t_{i}, y\left(t_{i}\right)\right)-f\left(t_{i}, 0\right)\right|+\left|f\left(t_{i}, 0\right)\right|\right]+\mid S_{i}^{*}\left(y\left(t_{i}\right) \mid\right]\right. \\
& +\sum_{k=1}^{p} \sum_{i=1}^{k} \frac{\lambda_{k}\left(\xi_{k}^{\rho}-t_{k}^{\rho}\right)^{\beta_{k}+1}}{\rho^{\beta_{k}+1} \Gamma\left(\beta_{k}+2\right)} \\
& \left.\times\left[{ }^{\rho} I_{t_{i-1}^{+}}^{\alpha_{i-1}-1}\left[\left|f\left(t_{i}, y\left(t_{i}\right)\right)-f\left(t_{i}, 0\right)\right|+\left|f\left(t_{i}, 0\right)\right|\right]+\left|S_{i}^{*}\left(y\left(t_{i}\right)\right)\right|\right]+|\eta|\right\} \\
\leq & \left(L_{1}\|r\|+M_{1} \|\right)\left\{\frac{t_{1}^{\rho \alpha_{0}}}{\rho^{\alpha_{0}} \Gamma\left(\alpha_{0}+1\right)}+\frac{1}{|\Omega|}\left\{\sum_{k=0}^{p} \frac{\lambda_{k}\left(\xi_{k}^{\rho}-t_{k}^{\rho}\right)^{\alpha_{k}+\beta_{k}}}{\rho^{\alpha_{k}+\beta_{k}} \Gamma\left(\alpha_{k}+\beta_{k}+1\right)}\right.\right. \\
& +\sum_{k=1}^{p} \sum_{i=1}^{k} \frac{\lambda_{k}\left(\xi_{k}^{\rho}-t_{k}^{\rho}\right)^{\beta_{k}}\left(t_{i}^{\rho}-t_{i-1}^{\rho}\right)^{\alpha_{i-1}}}{\rho_{k}+\alpha_{i-1} \Gamma\left(\beta_{k}+1\right) \Gamma\left(\alpha_{i-1}+1\right)} \\
+ & \sum_{k=2}^{p} \sum_{i=1}^{k-1} \frac{\lambda_{k}\left(\xi_{k}^{\rho}-t_{k}^{\rho}\right)^{\beta_{k}}\left(t_{k}^{\rho}-t_{i}^{\rho}\right)\left(t_{i}^{\rho}-t_{i-1}^{\rho}\right)^{\alpha_{i-1}-1}}{\rho^{\beta_{k}+\alpha_{i-1}} \Gamma\left(\beta_{k}+1\right) \Gamma\left(\alpha_{i-1}\right)} \\
& \left.\left.+\sum_{k=1}^{p} \sum_{i=1}^{k} \frac{\lambda_{k}\left(\xi_{k}^{\rho}-t_{k}^{\rho}\right)^{\beta_{k}+1}\left(t_{i}^{\rho}-t_{i-1}^{\rho}\right)^{\alpha_{i-1}-1}}{\rho^{\beta_{k}+\alpha_{i-1}} \Gamma\left(\beta_{k}+2\right) \Gamma\left(\alpha_{i-1}\right)}\right\}\right\}+\frac{M_{2}}{|\Omega|}\left\{\sum_{k=1}^{p} \sum_{i=1}^{k} \frac{\lambda_{k}\left(\xi_{k}^{\rho}-t_{k}^{\rho}\right)^{\beta_{k}}}{\rho^{\beta_{k}} \Gamma\left(\beta_{k}+1\right)}\right\} \\
& +\frac{M_{3}}{|\Omega|}\left\{\sum_{k=2}^{p} \sum_{i=1}^{k-1} \frac{\lambda_{k}\left(\xi_{k}^{\rho}-t_{k}^{\rho}\right)^{\beta_{k}}\left(t_{k}^{\rho}-t_{i}^{\rho}\right)}{\rho^{\beta_{k}+1} \Gamma\left(\beta_{k}+1\right)}+\sum_{k=1}^{p} \sum_{i=1}^{k} \frac{\lambda_{k}\left(\xi_{k}^{\rho}-t_{k}^{\rho}\right)^{\beta_{k}+1}}{\rho^{\beta_{k}+1} \Gamma\left(\beta_{k}+2\right)}\right\}+\frac{|\eta|}{|\Omega|} \\
\leq & \left(L_{1}\|r\|+M_{1} \|\right)\left\{\Lambda_{1}+\Lambda_{2}\right\}+M_{2} \Lambda_{3}+M_{3} \Lambda_{4}+\frac{|\eta|}{|\Omega|}<r,
\end{aligned}
$$

which, upon taking norm for $t \in J_{0}$, implies that $\|(F y)\|<r$. For $y \in B_{r}$ and $t \in J_{k}$, we have

$$
\begin{aligned}
|(F y)(t)| & \mid \\
= & \mid{ }^{\rho} I_{k}^{\alpha_{k}} f(t, y(t))+\sum_{i=1}^{k}\left[{ }^{\rho} I_{t_{i-1}^{+}}^{\alpha_{i-1}} f\left(t_{i}, y\left(t_{i}\right)\right)+S_{i}\left(y\left(t_{i}\right)\right)\right] \\
& +\sum_{i=1}^{k-1}\left(\frac{t_{k}^{\rho}-t_{i}^{\rho}}{\rho}\right)\left[{ }^{\rho} I_{t_{i-1}^{+}}^{\alpha_{i-1}-1} f\left(t_{i}, y\left(t_{i}\right)\right)+S_{i}^{*}\left(y\left(t_{i}\right)\right)\right] \\
& +\sum_{i=1}^{k}\left(\frac{t^{\rho}-t_{k}^{\rho}}{\rho}\right)\left[{ }^{\rho} I_{t_{i-1}}^{\alpha_{i-1}-1} f\left(t_{i}, y\left(t_{i}\right)\right)+S_{i}^{*}\left(y\left(t_{i}\right)\right)\right]+A \mid \\
\leq & { }_{t_{k}^{+}}^{\alpha_{k}}[|f(t, y(t))-f(t, 0)|+|f(t, 0)|] \\
& +\sum_{i=1}^{k}\left[{ }^{\rho} I_{t_{i-1}^{\alpha_{i-1}}}^{\alpha^{+}}\left[\left|f\left(t_{i}, y\left(t_{i}\right)\right)-f\left(t_{i}, 0\right)\right|+\left|f\left(t_{i}, 0\right)\right|\right]+\left|S_{i}\left(y\left(t_{i}\right)\right)\right|\right] \\
& +\sum_{i=1}^{k-1}\left(\frac{t_{k}^{\rho}-t_{i}^{\rho}}{\rho}\right) \\
& \times\left[{ }^{\rho} I_{t_{i-1}}^{\alpha_{i-1}-1}\left[\left|f\left(t_{i}, y\left(t_{i}\right)\right)-f\left(t_{i}, 0\right)\right|+\left|f\left(t_{i}, 0\right)\right|\right]+\left|S_{i}^{*}\left(y\left(t_{i}\right)\right)\right|\right] \\
& +\sum_{i=1}^{k}\left(\frac{t^{\rho}-t_{k}^{\rho}}{\rho}\right)
\end{aligned}
$$




$$
\begin{aligned}
& \times\left[{ }^{\rho} I_{t_{i-1}^{i-1}}^{\alpha_{i-1}}\left[\left|f\left(t_{i}, y\left(t_{i}\right)\right)-f\left(t_{i}, 0\right)\right|+\left|f\left(t_{i}, 0\right)\right|\right]+\left|S_{i}^{*}\left(y\left(t_{i}\right)\right)\right|\right] \\
& +\frac{1}{|\Omega|}\left\{\sum_{k=0}^{p} \lambda_{k}^{\rho} I_{t_{k}^{+}}^{\alpha_{k}+\beta_{k}}\left[\left|f\left(\xi_{k}, y\left(\xi_{k}\right)\right)-f\left(\xi_{k}, 0\right)\right|+\left|f\left(\xi_{k}, 0\right)\right|\right]\right. \\
& +\sum_{k=1}^{p} \sum_{i=1}^{k} \frac{\lambda_{k}\left(\xi_{k}^{\rho}-t_{k}^{\rho}\right)^{\beta_{k}}}{\rho^{\beta_{k}} \Gamma\left(\beta_{k}+1\right)}\left[{ }^{\rho} I_{t_{i-1}^{+}}^{\alpha_{i-1}}\left[\left|f\left(t_{i}, y\left(t_{i}\right)\right)-f\left(t_{i}, 0\right)\right|+\left|f\left(t_{i}, 0\right)\right|\right]+\mid S_{i}\left(y\left(t_{i}\right) \mid\right]\right. \\
& +\sum_{k=2}^{p} \sum_{i=1}^{k-1} \frac{\lambda_{k}\left(\xi_{k}^{\rho}-t_{k}^{\rho}\right)^{\beta_{k}}\left(t_{k}^{\rho}-t_{i}^{\rho}\right)}{\rho^{\beta_{k}+1} \Gamma\left(\beta_{k}+1\right)} \\
& \times\left[{ }^{\rho} I_{t_{i-1}^{+}}^{\alpha_{i-1}-1}\left[\left|f\left(t_{i}, y\left(t_{i}\right)\right)-f\left(t_{i}, 0\right)\right|+\left|f\left(t_{i}, 0\right)\right|\right]+\mid S_{i}^{*}\left(y\left(t_{i}\right) \mid\right]\right. \\
& +\sum_{k=1}^{p} \sum_{i=1}^{k} \frac{\lambda_{k}\left(\xi_{k}^{\rho}-t_{k}^{\rho}\right)^{\beta_{k}+1}}{\rho^{\beta_{k}+1} \Gamma\left(\beta_{k}+2\right)} \\
& \left.\times\left[{ }^{\rho} I_{t_{i-1}^{+}}^{\alpha_{i-1}-1}\left[\left|f\left(t_{i}, y\left(t_{i}\right)\right)-f\left(t_{i}, 0\right)\right|+\left|f\left(t_{i}, 0\right)\right|\right]+\left|S_{i}^{*}\left(y\left(t_{i}\right)\right)\right|\right]+|\eta|\right\} \\
& \leq\left(L_{1}\|r\|+M_{1} \|\right)\left\{\frac{\left(t_{k+1}^{\rho}-t_{k}^{\rho}\right)^{\alpha_{k}}}{\rho^{\alpha_{k}} \Gamma\left(\alpha_{k}+1\right)}+\sum_{i=1}^{k} \frac{\left(t_{i}^{\rho}-t_{i-1}^{\rho}\right)^{\alpha_{i-1}}}{\rho^{\alpha_{i-1}} \Gamma\left(\alpha_{i-1}+1\right)}\right. \\
& +\sum_{i=1}^{k-1}\left(\frac{t_{k}^{\rho}-t_{i}^{\rho}}{\rho}\right)\left(\frac{\left(t_{i}^{\rho}-t_{i-1}^{\rho}\right)^{\alpha_{i-1}-1}}{\rho^{\alpha_{i-1}-1} \Gamma\left(\alpha_{i-1}\right)}\right) \\
& +\sum_{i=1}^{k}\left(\frac{t_{k+1}^{\rho}-t_{k}^{\rho}}{\rho}\right)\left(\frac{\left(t_{i}^{\rho}-t_{i-1}^{\rho}\right)^{\alpha_{i-1}-1}}{\rho^{\alpha_{i-1}-1} \Gamma\left(\alpha_{i-1}\right)}\right)+\frac{1}{|\Omega|}\left\{\sum_{k=0}^{p} \frac{\lambda_{k}\left(\xi_{k}^{\rho}-t_{k}^{\rho}\right)^{\alpha_{k}+\beta_{k}}}{\rho^{\alpha_{k}+\beta_{k}} \Gamma\left(\alpha_{k}+\beta_{k}+1\right)}\right. \\
& +\sum_{k=1}^{p} \sum_{i=1}^{k} \frac{\lambda_{k}\left(\xi_{k}^{\rho}-t_{k}^{\rho}\right)^{\beta_{k}}\left(t_{i}^{\rho}-t_{i-1}^{\rho}\right)^{\alpha_{i-1}}}{\rho^{\beta_{k}+\alpha_{i-1}} \Gamma\left(\beta_{k}+1\right) \Gamma\left(\alpha_{i-1}+1\right)} \\
& +\sum_{k=2}^{p} \sum_{i=1}^{k-1} \frac{\lambda_{k}\left(\xi_{k}^{\rho}-t_{k}^{\rho}\right)^{\beta_{k}}\left(t_{k}^{\rho}-t_{i}^{\rho}\right)\left(t_{i}^{\rho}-t_{i-1}^{\rho}\right)^{\alpha_{i-1}-1}}{\rho^{\beta_{k}+\alpha_{i-1}} \Gamma\left(\beta_{k}+1\right) \Gamma\left(\alpha_{i-1}\right)} \\
& \left.\left.+\sum_{k=1}^{p} \sum_{i=1}^{k} \frac{\lambda_{k}\left(\xi_{k}^{\rho}-t_{k}^{\rho}\right)^{\beta_{k}+1}\left(t_{i}^{\rho}-t_{i-1}^{\rho}\right)^{\alpha_{i-1}-1}}{\rho^{\beta_{k}+\alpha_{i-1}} \Gamma\left(\beta_{k}+2\right) \Gamma\left(\alpha_{i-1}\right)}\right\}\right\} \\
& +M_{2}\left\{k+\frac{1}{|\Omega|} \sum_{k=1}^{p} \sum_{i=1}^{k} \frac{\lambda_{k}\left(\xi_{k}^{\rho}-t_{k}^{\rho}\right)^{\beta_{k}}}{\rho^{\beta_{k}} \Gamma\left(\beta_{k}+1\right)}\right\} \\
& +M_{3}\left[\sum_{i=1}^{k-1}\left(\frac{t_{k}^{\rho}-t_{i}^{\rho}}{\rho}\right)+\sum_{i=1}^{k}\left(\frac{t_{k+1}^{\rho}-t_{k}^{\rho}}{\rho}\right)+\frac{1}{|\Omega|}\left\{\sum_{k=2}^{p} \sum_{i=1}^{k-1} \frac{\lambda_{k}\left(\xi_{k}^{\rho}-t_{k}^{\rho}\right)^{\beta_{k}}\left(t_{k}^{\rho}-t_{i}^{\rho}\right)}{\rho^{\beta_{k}+1} \Gamma\left(\beta_{k}+1\right)}\right.\right. \\
& \left.\left.+\sum_{k=1}^{p} \sum_{i=1}^{k} \frac{\lambda_{k}\left(\xi_{k}^{\rho}-t_{k}^{\rho}\right)^{\beta_{k}+1}}{\rho^{\beta_{k}+1} \Gamma\left(\beta_{k}+2\right)}\right\}\right]+\frac{|\eta|}{|\Omega|} \\
& \leq\left(L_{1}\|r\|+M_{1} \|\right)\left\{\Lambda_{1}+\Lambda_{2}\right\}+M_{2} \Lambda_{3}+M_{3} \Lambda_{4}+\frac{|\eta|}{|\Omega|}<r .
\end{aligned}
$$

Consequently, we get $\|F y\|<r$ for $t \in J_{k}, k=0,1, \ldots, p$. Thus $F B_{r} \subset B_{r}$. 
Now, for $y, z \in \mathcal{G}$ and $t \in J_{0}$, we have

$$
\begin{aligned}
\mid(F y)( & t)-(F z)(t) \mid \\
\leq & { }^{\rho}{0^{+}}^{\alpha_{0}}|f(t, y(t))-f(t, z(t))|+\frac{1}{|\Omega|}\left\{\sum_{k=0}^{p} \lambda_{k}{ }^{\rho} I_{t_{k}^{+}}^{\alpha_{k}+\beta_{k}}\left|f\left(\xi_{k}, y\left(\xi_{k}\right)\right)-f\left(\xi_{k}, z\left(\xi_{i}\right)\right)\right|\right. \\
& +\sum_{k=1}^{p} \sum_{i=1}^{k} \frac{\lambda_{k}\left(\xi_{k}^{\rho}-t_{k}^{\rho}\right)^{\beta_{k}}}{\rho^{\beta_{k}} \Gamma\left(\beta_{k}+1\right)}\left[{ }^{\rho} I_{t_{i-1}^{+}}^{\alpha_{i-1}}\left|f\left(t_{i}, y\left(t_{i}\right)\right)-f\left(t_{i}, z\left(\xi_{i}\right)\right)\right|+\left|S_{i}\left(y\left(t_{i}\right)\right)-S_{i}\left(z\left(t_{i}\right)\right)\right|\right] \\
& +\sum_{k=2}^{p} \sum_{i=1}^{k-1} \frac{\lambda_{k}\left(\xi_{k}^{\rho}-t_{k}^{\rho}\right)^{\beta_{k}}\left(t_{k}^{\rho}-t_{i}^{\rho}\right)}{\rho^{\beta_{k}+1} \Gamma\left(\beta_{k}+1\right)} \\
& \times\left[{ }^{\rho} I_{t_{i-1}^{+}}^{\alpha_{i-1}-1}\left|f\left(t_{i}, y\left(t_{i}\right)\right)-f\left(t_{i}, z\left(t_{i}\right)\right)\right|+\left|S_{i}^{*}\left(y\left(t_{i}\right)\right)-S_{i}^{*}\left(z\left(t_{i}\right)\right)\right|\right] \\
& +\sum_{k=1}^{p} \sum_{i=1}^{k} \frac{\lambda_{k}\left(\xi_{k}^{\rho}-t_{k}^{\rho}\right)^{\beta_{k}+1}}{\rho^{\beta_{k}+1} \Gamma\left(\beta_{k}+2\right)} \\
& \left.\times\left[{ }^{\rho} I_{t_{i-1}^{+} \alpha_{i-1}-1}\left|f\left(t_{i}, y\left(t_{i}\right)\right)-f\left(t_{i}, z\left(t_{i}\right)\right)\right|+\left|S_{i}^{*}\left(y\left(t_{i}\right)\right)-S^{*}\left(z\left(t_{i}\right)\right)\right|\right]\right\} \\
\leq & \left\{L_{1}\left(\Lambda_{1}+\Lambda_{2}\right)+L_{2} \Lambda_{3}+L_{3} \Lambda_{4}\right\}\|x-y\| .
\end{aligned}
$$

In a similar way for $t \in J_{k}$, we obtain

$$
\begin{aligned}
& |(F y)(t)-(F z)(t)| \\
& \leq^{\rho} I_{t_{k}^{+}}^{\alpha_{k}}|f(t, y(t))-f(t, z(t))| \\
& +\sum_{i=1}^{k}\left[{ }^{\rho} I_{t_{i-1}^{+}}^{\alpha_{i-1}}\left|f\left(t_{i}, y\left(t_{i}\right)\right)-f\left(t_{i}, z\left(t_{i}\right)\right)\right|+\left|S_{i}\left(y\left(t_{i}\right)\right)-S_{i}\left(z\left(t_{i}\right)\right)\right|\right] \\
& +\sum_{i=1}^{k-1}\left(\frac{t_{k}^{\rho}-t_{i}^{\rho}}{\rho}\right)\left[{ }^{\rho} I_{t_{i-1}^{+}}^{\alpha_{i-1}-1}\left|f\left(t_{i}, y\left(t_{i}\right)\right)-f\left(t_{i}, z\left(t_{i}\right)\right)\right|+\left|S_{i}^{*}\left(y\left(t_{i}\right)\right)-S_{i}^{*}\left(z\left(t_{i}\right)\right)\right|\right] \\
& +\sum_{i=1}^{k}\left(\frac{t^{\rho}-t_{k}^{\rho}}{\rho}\right)\left[{ }^{\rho} I_{t_{i-1}^{+}}^{\alpha_{i-1}-1}\left|f\left(t_{i}, y\left(t_{i}\right)\right)-f\left(t_{i}, z\left(t_{i}\right)\right)\right|+\left|S_{i}^{*}\left(y\left(t_{i}\right)\right)-S_{i}^{*}\left(z\left(t_{i}\right)\right)\right|\right] \\
& +\frac{1}{|\Omega|}\left\{\sum_{k=0}^{p} \lambda_{k}{ }^{\rho} I_{t_{k}^{+}}^{\alpha_{k}+\beta_{k}}\left|f\left(\xi_{k}, y\left(\xi_{k}\right)\right)-f\left(\xi_{k}, z\left(\xi_{i}\right)\right)\right|\right. \\
& +\sum_{k=1}^{p} \sum_{i=1}^{k} \frac{\lambda_{k}\left(\xi_{k}^{\rho}-t_{k}^{\rho}\right)^{\beta_{k}}}{\rho^{\beta_{k}} \Gamma\left(\beta_{k}+1\right)}\left[{ }^{\rho} I_{t_{i-1}^{+}}^{\alpha_{i-1}}\left|f\left(t_{i}, y\left(t_{i}\right)\right)-f\left(t_{i}, z\left(\xi_{i}\right)\right)\right|+\left|S_{i}\left(y\left(t_{i}\right)\right)-S_{i}\left(z\left(t_{i}\right)\right)\right|\right] \\
& +\sum_{k=2}^{p} \sum_{i=1}^{k-1} \frac{\lambda_{k}\left(\xi_{k}^{\rho}-t_{k}^{\rho}\right)^{\beta_{k}}\left(t_{k}^{\rho}-t_{i}^{\rho}\right)}{\rho^{\beta_{k}+1} \Gamma\left(\beta_{k}+1\right)} \\
& \times\left[{ }^{\rho} I_{t_{i-1}^{+}}^{\alpha_{i-1}-1}\left|f\left(t_{i}, y\left(t_{i}\right)\right)-f\left(t_{i}, z\left(t_{i}\right)\right)\right|+\left|S_{i}^{*}\left(y\left(t_{i}\right)\right)-S_{i}^{*}\left(z\left(t_{i}\right)\right)\right|\right] \\
& +\sum_{k=1}^{p} \sum_{i=1}^{k} \frac{\lambda_{k}\left(\xi_{k}^{\rho}-t_{k}^{\rho}\right)^{\beta_{k}+1}}{\rho^{\beta_{k}+1} \Gamma\left(\beta_{k}+2\right)}
\end{aligned}
$$




$$
\begin{aligned}
& \left.\times\left[{ }^{\rho} I_{t_{i-1}^{+}}^{\alpha_{i-1}-1}\left|f\left(t_{i}, y\left(t_{i}\right)\right)-f\left(t_{i}, z\left(t_{i}\right)\right)\right|+\left|S_{i}^{*}\left(y\left(t_{i}\right)\right)-S^{*}\left(z\left(t_{i}\right)\right)\right|\right]\right\} \\
\leq & \left\{L_{1}\left(\Lambda_{1}+\Lambda_{2}\right)+L_{2} \Lambda_{3}+L_{3} \Lambda_{4}\right\}\|y-z\| .
\end{aligned}
$$

Consequently, we obtain

$$
\|F y-F z\| \leq\left\{L_{1}\left(\Lambda_{1}+\Lambda_{2}\right)+L_{2} \Lambda_{3}+L_{3} \Lambda_{4}\right\}\|y-z\|, \quad t \in J_{k}, k=0,1,2, \ldots, p,
$$

which, in view of (3.7), implies that $F$ is a contraction. Thus the conclusion of the theorem follows by the contraction mapping principle.

Example 3.4 With $\rho=1 / 2, \alpha_{0}=5 / 4, \alpha_{1}=7 / 4, \beta_{0}=1 / 2, \beta_{1}=3 / 2, \lambda_{0}=1 / 3, \lambda_{1}=1 / 4, \xi_{0}=$ $1 / 2, \xi_{1}=3 / 2, t_{1}=3 / 4$, we consider the problem

$$
\left\{\begin{array}{l}
{ }_{c}^{\rho} D_{t_{k}^{+}}^{\alpha_{k}} y(t)=\frac{1}{(t+9)^{2}}\left(\frac{|y(t)|+2}{|y(t)|+1}+\cos t\right), \quad t \in[0,2], t \neq 3 / 4, k=0,1, \\
\Delta y(3 / 4)=\frac{|y(3 / 4)|}{12+|y(3 / 4)|}, \quad \Delta \delta y(3 / 4)=\frac{|y(3 / 4)|}{9+|y(3 / 4)|}, \quad k=1,2, \ldots, p, \\
u(0)=\sum_{k=0}^{1} \lambda_{k^{\rho}} I_{t_{k}^{+}}^{\beta_{k}} y\left(\xi_{k}\right)-1, \quad \delta y(0)=0 .
\end{array}\right.
$$

Using the given data, we find that $|\Omega| \approx 0.438425, \Lambda_{1} \approx 12.512411, \Lambda_{2} \approx 1.442181$, $\Lambda_{3} \approx 1.260667, \Lambda_{4} \approx 2.903232$, where $\Omega, \Lambda_{1}, \Lambda_{2}, \Lambda_{3}$ and $\Lambda_{4}$ are given by (2.10), (3.2), (3.3), (3.4), and (3.5), respectively. Clearly, all the assumptions of Theorem 3.2 hold with $L_{2}=$ $1 / 12, L_{3}=1 / 9, M_{2}=M_{3}=1, \phi(t)=\frac{2+\cos t}{(t+9)^{2}}$, and $p=1$. Also $L_{2} \Lambda_{3}+L_{3} \Lambda_{4} \approx 0.4276369325<1$. Therefore, by Theorem 3.2, we deduce that the impulsive integro-initial value problem (3.8) has at least one solution on [0,2]. Furthermore, the hypothesis of Theorem 3.3 is satisfied with $L_{1}=1 / 81, L_{2}=1 / 12, L_{3}=1 / 9, M_{2}=M_{3}=1$. Moreover, $L_{1}\left(\Lambda_{1}+\Lambda_{2}\right)+L_{2} \Lambda_{3}+$ $L_{3} \Lambda_{4} \approx 0.599915849<1$. So, Theorem 3.3 implies that the impulsive integro-initial value problem (3.8) has a unique solution on $[0,2]$.

\section{Extremal solutions}

Here we discuss the existence of extremal solutions for problem (1.1). Before presenting the main result, we define lower and upper solutions for the problem at hand and prove a new comparison result.

Definition 4.1 Function $y(t)$ is said to be a lower solution of problem (1.1) if

$$
\left\{\begin{array}{l}
{ }_{c}^{\rho} D_{t_{k}^{+}}^{\alpha_{k}} y(t) \leq f(t, y(t)), \quad 1<\alpha_{k} \leq 2, k=0,1,2, \ldots, p, t \in J^{\prime}, \\
\triangle y\left(t_{k}\right) \leq S_{k}\left(y\left(t_{k}\right)\right), \quad \Delta \delta y\left(t_{k}\right) \leq S_{k}^{*}\left(y\left(t_{k}\right)\right), \quad k=1,2, \ldots, p, \\
y(0) \leq \sum_{k=0}^{p} \lambda_{k}^{\rho} I_{t_{k}^{+}}^{\beta_{k}} y\left(\xi_{k}\right)+\eta, \quad \delta y(0)=0, \quad t_{k}<\xi_{k}<t_{k+1} .
\end{array}\right.
$$

By reversing the inequalities in the above definition, we obtain the corresponding definition of an upper solution of (1.1). 
Lemma 4.2 (Comparison result) If $\sum_{k=0}^{p} \frac{\lambda_{k}\left(\xi_{k}^{\rho}-t_{k}^{\rho}\right)^{\beta_{k}}}{\rho^{\beta_{k}} \Gamma\left(\beta_{k}+1\right)}<1$ and $y \in \mathcal{E}=P C_{\delta}^{1}(J, \mathbb{R}) \cap A C_{\delta}^{2}\left(J_{k}, \mathbb{R}\right)$ satisfies

$$
\left\{\begin{array}{l}
{ }_{c}^{\rho} D_{t_{k}^{+}}^{\alpha_{k}} y(t) \geq 0, \quad 0<\alpha_{k} \leq 2, k=0,1,2, \ldots, p, t \in J^{\prime}, \\
\triangle y\left(t_{k}\right) \geq 0, \quad \Delta \delta y\left(t_{k}\right) \geq 0, \quad k=1,2, \ldots, p, \\
y(0) \geq \sum_{k=0}^{p} \lambda_{k}^{\rho} I_{t_{k}^{+}}^{\beta_{k}} y\left(\xi_{k}\right), \quad \delta y(0)=0, \quad t_{k}<\xi_{k}<t_{k+1},
\end{array}\right.
$$

then $y(t) \geq 0, \forall t \in J$.

Proof Consider a modified form of problem (2.11) given by

$$
\left\{\begin{array}{l}
{ }_{c}^{\rho} D_{t_{k}^{+}}^{\alpha_{k}} y(t)=g(t), \quad 0<\alpha_{k} \leq 2, k=0,1,2, \ldots, p, t \in J^{\prime}, \\
\triangle y\left(t_{k}\right)=S_{k}, \quad \Delta \delta y\left(t_{k}\right)=S_{k}^{*}, \quad k=1,2, \ldots, p, \\
y(0)=\sum_{k=0}^{p} \lambda_{k}^{\rho} I_{t_{k}^{+}}^{\beta_{k}} y\left(\xi_{k}\right)+\eta, \quad \delta y(0)=0, \quad t_{k}<\xi_{k}<t_{k+1},
\end{array}\right.
$$

where $g(t) \in C\left(J, \mathbb{R}^{+}\right)$and $S_{k}, S_{k}^{*}(k=1,2, \ldots, p), \eta$ are nonnegative constants.

Then the solution of problem (4.3) is

$$
y(t)=\left\{\begin{array}{l}
{ }^{\rho} I_{0^{+}}^{\alpha_{0}} g(t)+\sigma, \quad t \in J_{0}, \\
{ }^{\rho} I_{t_{k}^{+}}^{\alpha_{k}} g(t)+\sum_{i=1}^{k}\left[{ }^{\rho} I_{t_{i-1}^{+}}^{\alpha_{i-1}} g\left(t_{i}\right)+S_{i}\right]+\sum_{i=1}^{k-1}\left(\frac{t_{k}^{\rho}-t_{i}^{\rho}}{\rho}\right)\left[{ }^{\rho} I_{t_{i-1}^{+}}^{\alpha_{i-1}-1} g\left(t_{i}\right)+S_{i}^{*}\right] \\
\left.\quad+\sum_{i=1}^{k} \frac{t^{\rho}-t_{k}^{\rho}}{\rho}\right)\left[{ }^{\rho} I_{t_{i-1}^{+}}^{\alpha_{i-1}-1} g\left(t_{i}\right)+S_{i}^{*}\right]+\sigma, \\
\quad t \in J_{k}, k=1,2, \ldots, p,
\end{array}\right.
$$

where

$$
\begin{aligned}
\sigma= & \frac{1}{\Omega}\left\{\sum_{k=0}^{p} \lambda_{k}{ }^{\rho} I_{t_{k}^{+}}^{\alpha_{k}+\beta_{k}} g\left(\xi_{k}\right)+\sum_{k=1}^{p} \sum_{i=1}^{k} \frac{\lambda_{k}\left(\xi_{k}^{\rho}-t_{k}^{\rho}\right)^{\beta_{k}}}{\rho^{\beta_{k}} \Gamma\left(\beta_{k}+1\right)}\left[{ }^{\rho} I_{t_{i-1}}^{\alpha_{i-1}} g\left(t_{i}\right)+S_{i}\right]\right. \\
& +\sum_{k=2}^{p} \sum_{i=1}^{k-1} \frac{\lambda_{k}\left(\xi_{k}^{\rho}-t_{k}^{\rho}\right)^{\beta_{k}}\left(t_{k}^{\rho}-t_{i}^{\rho}\right)}{\rho^{\beta_{k}+1} \Gamma\left(\beta_{k}+1\right)}\left[{ }^{\rho} I_{t_{i-1}^{+}}^{\alpha_{i-1}-1} g\left(t_{i}\right)+S_{i}^{*}\right] \\
& \left.+\sum_{k=1}^{p} \sum_{i=1}^{k} \frac{\lambda_{k}\left(\xi_{k}^{\rho}-t_{k}^{\rho}\right)^{\beta_{k}+1}}{\rho^{\beta_{k}+1} \Gamma\left(\beta_{k}+2\right)}\left[{ }^{\rho} I_{t_{i-1}^{+}}^{\alpha_{i-1}-1} g\left(t_{i}\right)+S_{i}^{*}\right]+\eta\right\} .
\end{aligned}
$$

In view of the nonnegative nature of the function $g(t)$ and constants $S_{k}, S_{k}^{*}, \eta$, the conclusion of Lemma 4.2 follows from (4.4).

Our next result, dealing with the extremal solutions of (1.1), relies on the following fixed point theorem [34].

Lemma 4.3 Let $[a, b]$ be a nonempty order interval of a subset $Y$ of an ordered Banach space $X$ and let $P:[a, b] \rightarrow[a, b]$ be a nondecreasing mapping. If each sequence $\left\{P y_{n}\right\} \subset$ $P([a, b])$ converges whenever $\left\{y_{n}\right\}$ is a monotone sequence in $[a, b]$, then the sequence of $P$ iterates of a converges to the least fixed point $y_{*}$ of $P$ and the sequence of P-iterates of $b$ 
converges to the greatest fixed point $y^{*}$ of P. Moreover,

$$
y_{*}=\min \{x \in[a, b]: x \geq P x\}, \quad y^{*}=\max \{x \in[a, b]: x \leq P x\} .
$$

\section{Theorem 4.4 Assume that}

$\left(A_{1}\right)$ the functions $f(t, y), S_{k}(y), S_{k}^{*}(y), k=1, \ldots, p$, are continuous and nondecreasing in $y$;

$\left(A_{2}\right)$ there exist lower and upper solutions $y_{0}$ and $z_{0} \in \mathcal{E}$ for problem (1.1), respectively, such that $y_{0} \leq z_{0}$;

(A $\left.A_{3}\right) \sum_{k=0}^{p} \frac{\lambda_{k}\left(\xi_{k}^{\rho}-t_{k}^{\rho}\right)_{k}}{\rho^{\beta} \beta_{k}\left(\beta_{k}+1\right)}<1$.

Then problem (1.1) has extremal solutions in the sector $\left[y_{0}, z_{0}\right]$.

Proof Consider problem (2.11) with $h(t)=f(t, v(t)), S_{k}=S_{k}\left(v\left(t_{k}\right)\right)$ and $S_{k}^{*}=S_{k}^{*}\left(v\left(t_{k}\right)\right), k=$ $1,2, \ldots, p$. Let us consider the operator $F$ defined by (3.1) from $\left[y_{0}, z_{0}\right]$ to $\mathcal{E}$ such that $y(t)=$ $F v(t)$. First, it will be shown that $F$ maps $\left[y_{0}, z_{0}\right]$ into $\left[y_{0}, z_{0}\right]$.

Let $y_{1}=F y_{0}, z_{1}=F z_{0}$. Then $y_{1}, z_{1}$ are well defined and respectively satisfy the problems

$$
\left\{\begin{array}{l}
{ }_{c}^{\rho} D_{t_{k}^{+}}^{\alpha_{k}} y_{1}(t)=f\left(t, y_{0}(t)\right), \quad 1<\alpha_{k} \leq 2, k=0,1,2, \ldots, p, t \in J^{\prime}, \\
\triangle y_{1}\left(t_{k}\right)=S_{k}\left(y_{0}\left(t_{k}\right)\right), \quad \Delta \delta y_{1}\left(t_{k}\right)=S_{k}^{*}\left(y_{0}\left(t_{k}\right)\right), \quad k=1,2, \ldots, p, \\
y_{1}(0)=\sum_{k=0}^{p} \lambda_{k} I_{t_{k}^{+}}^{\beta_{k}} y_{1}\left(\xi_{k}\right)+\eta, \quad \delta y_{1}(0)=0, \quad t_{k}<\xi_{k}<t_{k+1}
\end{array}\right.
$$

and

$$
\left\{\begin{array}{l}
{ }_{c}^{\rho} D_{t_{k}^{+}}^{\alpha_{k}} z_{1}(t)=f\left(t, z_{0}(t)\right), \quad 1<\alpha_{k} \leq 2, k=0,1,2, \ldots, p, t \in J^{\prime}, \\
\triangle z_{1}\left(t_{k}\right)=S_{k}\left(z_{0}\left(t_{k}\right)\right), \quad \triangle \delta z_{1}\left(t_{k}\right)=S_{k}^{*}\left(z_{0}\left(t_{k}\right)\right), \quad k=1,2, \ldots, p, \\
z_{1}(0)=\sum_{k=0}^{p} \lambda_{k}{ }^{\rho} I_{t_{k}^{+}}^{\beta_{k}} z_{1}\left(\xi_{k}\right)+\eta, \quad \delta z_{1}(0)=0, \quad t_{k}<\xi_{k}<t_{k+1} .
\end{array}\right.
$$

Setting $u=y_{1}-y_{0}$ and using the definition of a lower solution, we get

$$
\left\{\begin{array}{l}
{ }_{c}^{\rho} D_{t_{k}^{+}}^{\alpha_{k}} u(t) \geq 0, \quad 1<\alpha_{k} \leq 2, k=0,1,2, \ldots, p, t \in J^{\prime}, \\
\triangle u\left(t_{k}\right) \geq 0, \quad \Delta \delta u\left(t_{k}\right) \geq 0, \quad k=1,2, \ldots, p, \\
u(0) \geq \sum_{k=0}^{p} \lambda_{k}^{\rho} I_{t_{k}^{+}}^{\beta_{k}} u\left(\xi_{k}\right), \quad \delta u(0)=0, \quad t_{k}<\xi_{k}<t_{k+1},
\end{array}\right.
$$

which, by Lemma 4.2, implies that $u(t) \geq 0, \forall t \in J$. Thus $F y_{0} \geq y_{0}$. Similarly, using the definition of an upper solution, one can show that $F z_{0} \leq z_{0}$.

Now, we define $\omega=z_{1}-y_{1}$ and use (4.6) and (4.7) together with assumption $A_{1}$ to obtain

$$
\left\{\begin{array}{l}
{ }_{c}^{\rho} D_{t_{k}^{+}}^{\alpha_{k}} \omega(t)=f\left(t, z_{0}(t)\right)-f\left(t, y_{0}(t)\right) \geq 0, \quad 1<\alpha_{k} \leq 2, k=0,1,2, \ldots, p, t \in J^{\prime}, \\
\triangle \omega\left(t_{k}\right)=S_{k}\left(z_{0}\left(t_{k}\right)\right)-S_{k}\left(y_{0}\left(t_{k}\right)\right) \geq 0, \quad k=1,2, \ldots, p, \\
\triangle \delta \omega\left(t_{k}\right)=S_{k}^{*}\left(z_{0}\left(t_{k}\right)\right)-S_{k}^{*}\left(y_{0}\left(t_{k}\right)\right) \geq 0, \quad k=1,2, \ldots, p, \\
\omega(0)=\sum_{k=0}^{p} \lambda_{k}{ }^{\rho} I_{t_{k}^{+}}^{\beta_{k}} \omega\left(\xi_{k}\right), \quad \delta \omega(0)=0, \quad t_{k}<\xi_{k}<t_{k+1} .
\end{array}\right.
$$

Applying Lemma 4.2 , we deduce that $\omega(t) \geq 0$, that is, $F z_{0} \geq F y_{0}$. Thus $F$ is nondecreasing and $y_{0} \leq F y \leq z_{0}$ for any $y \in\left[y_{0}, z_{0}\right]$. In consequence, $F\left[y_{0}, z_{0}\right] \subset\left[y_{0}, z_{0}\right]$ and $\|F y\| \leq \max \left\{\left\|y_{0}\right\|,\left\|z_{0}\right\|\right\}:=\Delta$. 
Let $\left\{y_{n}\right\}$ be a monotone sequence in $\left[y_{0}, z_{0}\right]$. Then $y_{0} \leq F y_{n} \leq z_{0}$ and $\left\|F y_{n}\right\| \leq \Delta$. Next we show that the sequence $\left\{F y_{n}\right\}$ is equicontinuous. For any $(t, y) \in J \times[-\Delta, \Delta]$, there exist positive constants $K_{1}, K_{2}$ such that $|f(t, y)| \leq K_{1},\left|S_{k}^{*}(y)\right| \leq K_{2}$. Then, for any $\tau_{1}, \tau_{2} \in J_{k}$ with $\tau_{1} \leq \tau_{2}, k=1,2, \ldots, p$, we obtain

$$
\begin{aligned}
& \left\|F(y)\left(\tau_{2}\right)-F(y)\left(\tau_{1}\right)\right\| \\
& =\| \frac{\rho^{1-\alpha_{k}}}{\Gamma\left(\alpha_{k}\right)}\left[\int_{t_{k}}^{\tau_{1}} s^{\rho-1}\left[\left(\tau_{2}^{\rho}-s^{\rho}\right)^{\alpha_{k}-1}-\left(\tau_{1}^{\rho}-s^{\rho}\right)^{\alpha_{k}-1}\right] f(s, y(s)) d s\right. \\
& \left.\quad+\int_{\tau_{1}}^{\tau_{2}} s^{\rho-1}\left(\tau_{2}^{\rho}-s^{\rho}\right)^{\alpha_{k}-1} f(s, y(s)) d s\right] \\
& \quad+\sum_{i=1}^{k}\left(\frac{\left(\tau_{2}^{\rho}-t_{k}^{\rho}\right)-\left(\tau_{1}^{\rho}-t_{k}^{\rho}\right)}{\rho}\right) \\
& \quad \times\left(\frac{\rho^{2-\alpha_{i}}}{\Gamma\left(\alpha_{i}-1\right)} \int_{t_{i-1}}^{t_{i}} s^{\rho-1}\left(t_{i}^{\rho}-s^{\rho}\right)^{\alpha_{i}-2} f(s, y(s)) d s+S_{i}^{*}\left(y\left(t_{i}\right)\right)\right) \| \\
& \leq \\
& \quad \frac{K_{1}}{\rho^{\alpha_{k}} \Gamma\left(\alpha_{k}+1\right)}\left\{2\left(\tau_{2}^{\rho}-\tau_{1}^{\rho}\right)^{\alpha_{k}}+\left|\left(\tau_{2}^{\rho}-t_{k}^{\rho}\right)^{\alpha_{k}}-\left(\tau_{1}^{\rho}-t_{k}^{\rho}\right)^{\alpha_{k}}\right|\right\} \\
& \quad+K_{1} \sum_{i=1}^{k}\left(\frac{\left[\left(\tau_{2}^{\rho}-t_{k}^{\rho}\right)-\left(\tau_{1}^{\rho}-t_{k}^{\rho}\right)\right]\left(t_{i}^{\rho}-t_{i-1}^{\rho}\right)^{\alpha_{i}-1}}{\rho^{\alpha_{i}} \Gamma\left(\alpha_{i}\right)}\right)+K_{2} \sum_{i=1}^{k}\left(\frac{\left(\tau_{2}^{\rho}-t_{k}^{\rho}\right)-\left(\tau_{1}^{\rho}-t_{k}^{\rho}\right)}{\rho}\right),
\end{aligned}
$$

which tends to zero as $\tau_{2}-\tau_{1} \rightarrow 0$ independent of $y$. A similar conclusion follows for $\tau_{1}, \tau_{2} \in J_{0}$. Thus, $\left\{F y_{n}\right\}$ is equicontinuous on all $J_{k}, 0 \leq k \leq p$. So $F$ is relatively compact on $\left[y_{0}, z_{0}\right]$. Hence, by the Arzelá-Ascoli theorem, $F$ is compact on $\left[y_{0}, z_{0}\right]$, and consequently $\left\{F y_{n}\right\}$ converges in $F\left(\left[y_{0}, z_{0}\right]\right)$. Thus all the hypotheses of Lemma 4.3 hold, and the conclusion of Lemma 4.3 implies that $F$ has the least and greatest fixed points in $\left[y_{0}, z_{0}\right]$. This shows that problem (1.1) has extremal solutions on $\left[y_{0}, z_{0}\right]$.

Example 4.5 Consider the problem

$$
\left\{\begin{array}{l}
{ }_{c}^{\rho} D_{t_{k}^{+}}^{\alpha_{k}} y(t)=\frac{t\left(t^{1 / 3}-(1 / 2)^{1 / 3}\right)^{2}}{1100}\left(1+(y(t))^{3}\right), \quad t \in[0,1], t \neq 1 / 2, k=0,1, \\
\Delta y\left(t_{1}\right)=\frac{1}{4} \tan ^{-1} y\left(t_{1}\right), \quad \Delta \delta y\left(t_{1}\right)=\frac{y\left(t_{1}\right)}{5}, \\
y(0)=\sum_{k=0}^{1} \lambda_{k}^{\rho} I_{t_{k}^{+}}^{\beta_{k}} y\left(\xi_{k}\right)+1 / 4, \quad \delta y(0)=0,
\end{array}\right.
$$

where $\rho=1 / 3, \alpha_{0}=5 / 4, \alpha_{1}=3 / 2, \beta_{0}=1 / 2, \beta_{1}=3 / 2, \lambda_{0}=1 / 10, \lambda_{1}=1 / 7, \xi_{0}=1 / 4, \xi_{1}=$ $3 / 4, t_{1}=1 / 2, f(t, y)=\frac{t\left(t^{1 / 3}-(1 / 2)^{1 / 3}\right)^{2}}{1100}\left(1+y^{3}\right), S_{1}(y)=\frac{1}{4} \tan ^{-1} y$, and $S_{1}^{*}(y)=\frac{y}{5}$.

We take $y_{0}(t)=0$ as the lower solution and

$$
z_{0}(t)= \begin{cases}1+\frac{t^{2 \rho}}{2 \rho^{2}}, & 0 \leq t \leq \frac{1}{2}, \\ 1+\frac{t^{2 \rho}}{\rho^{2}}, & \frac{1}{2}<t \leq 1\end{cases}
$$

as the upper solution of problem (4.10). With the given data, it is found that

$$
\sum_{k=0}^{1} \frac{\lambda_{k}\left(\xi_{k}^{\rho}-t_{k}^{\rho}\right)^{\beta_{k}}}{\rho^{\beta_{k}} \Gamma\left(\beta_{k}+1\right)}=0.1768586259<1 .
$$


Also, assumption $\left(A_{1}\right)$ is clearly satisfied. Thus, by Theorem 4.4, problem (4.10) has extremal solutions on $\left[y_{0}, z_{0}\right]$.

\section{Conclusions}

We have developed an existence theory for impulsive multi-order nonlinear Caputo-type generalized fractional differential equations equipped with nonlocal conditions involving Katugampola type generalized fractional integrals. The work presented in this paper is new and significantly contributes to the existing literature on the topic. By fixing the parameters involved in the problem, we can obtain some new results as special cases of those derived in this paper. For instance, our results correspond to those for nonlinear single order Caputo-type generalized fractional differential equations with generalized fractional integro-initial conditions if we set $\alpha_{k}=\alpha$. The results obtained in [26] appear as a special case of those established in Sect. 4 for $\rho=1$.

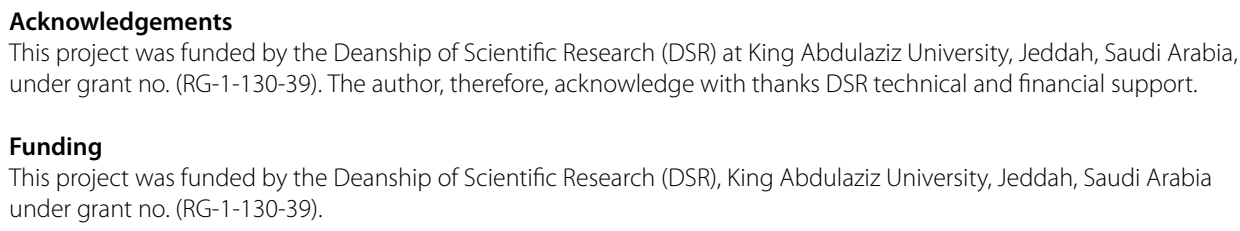

\section{Abbreviations}

Not applicable.

\section{Availability of data and materials}

Not applicable.

\section{Competing interests}

The authors declare that they have no competing interests.

\section{Authors' contributions}

Each of the authors, BA, MA, JJN, and AA contributed equally to each part of this work. All authors read and approved the final manuscript.

\section{Author details}

${ }^{1}$ Nonlinear Analysis and Applied Mathematics (NAAM)-Research Group, Department of Mathematics, Faculty of Science, King Abdulaziz University, Jeddah, Saudi Arabia. ²Departamento de Estatistica, Análise Matemática e Optimización, Instituto de Matematicas, University of Santiago de Compostela, Santiago de Compostela, Spain.

\section{Publisher's Note}

Springer Nature remains neutral with regard to jurisdictional claims in published maps and institutional affiliations.

Received: 6 March 2019 Accepted: 7 June 2019 Published online: 24 June 2019

\section{References}

1. Alur, R., Courcoubetis, C., Dill, D.: Model checking for real-time systems. In: Proc. 5th Ann. IEEE Symp. on Logic in Computer Science, Philadelphia, PA, pp. 414-425 (1990)

2. Lygeros, J., Godbole, D.N., Sastry, S.: A verified hybrid controller for automated vehicles. IEEE Trans. Autom. Control 43, 522-539 (1998)

3. Varaiya, P.: Smart cars on smart roads: problems of control. IEEE Trans. Autom. Control 38, 195-207 (1993)

4. Altafini, C., Speranzon, A., Johansson, K.H.: Hybrid control of a truck and trailer vehicle. In: Tomlin, C.J., Greenstreet, M.R. (eds.) Hybrid Systems: Computation and Control. Lecture Notes in Computer Science, vol. 2289. Springer, New York (2002)

5. Balluchi, A., Benvenutti, L., Di Benedetto, M., Pinello, C., Sangiovanni-Vincentelli, A.: Automotive engine control and hybrid systems: challenges and opportunities. Proc. IEEE 7, 888-912 (2000)

6. Wang, J.R., Zhou, Y., Feckan, M.: On recent developments in the theory of boundary value problems for impulsive fractional differential equations. Comput. Math. Appl. 64, 3008-3020 (2012)

7. Graef, J.R., Kong, L.: Existence of positive solutions to a higher order singular boundary value problem with fractional q-derivatives. Fract. Calc. Appl. Anal. 16, 695-708 (2013)

8. O'Regan, D., Stanek, S.: Fractional boundary value problems with singularities in space variables. Nonlinear Dyn. 71, 641-652 (2013) 
9. Henderson, J., Luca, R.: Nonexistence of positive solutions for a system of coupled fractional boundary value problems. Bound. Value Probl. 2015, 138 (2015)

10. Zhang, L., Ahmad, B., Wang, G.: Successive iterations for positive extremal solutions of nonlinear fractional differential equations on a half line. Bull. Aust. Math. Soc. 91, 116-128 (2015)

11. Ahmad, B., Alsaedi, A., Aljoudi, S., Ntouyas, S.K.: On a coupled system of sequential fractional differential equations with variable coefficients and coupled integral boundary conditions. Bull. Math. Soc. Sci. Math. Roum. 60(108), 3-18 (2017)

12. Wang, G., Pei, K., Agarwal, R.P., Zhang, L., Ahmad, B.: Nonlocal Hadamard fractional boundary value problem with Hadamard integral and discrete boundary conditions on a half-line. J. Comput. Appl. Math. 343, 230-239 (2018)

13. Ahmad, B., Luca, R.: Existence of solutions for a system of fractional differential equations with coupled nonlocal boundary conditions. Fract. Calc. Appl. Anal. 21,423-441 (2018)

14. Henderson, J., Luca, R.: Positive solutions for a system of coupled fractional boundary value problems. Lith. Math. J. 58, 15-32 (2018)

15. Ding, X., Nieto, J.J.: Analytical solutions for multi-term time-space fractional partial differential equations with nonlocal damping terms. Fract. Calc. Appl. Anal. 21, 312-335 (2018)

16. Agarwal, R.P., Ahmad, B., Alsaedi, A.: Fractional-order differential equations with anti-periodic boundary conditions: a survey. Bound. Value Probl. 2015, 138 (2017)

17. Ahmad, B., Alghanmi, M., Ntouyas, S.K., Alsaedi, A.: Fractional differential equations involving generalized derivative with Stieltjes and fractional integral boundary conditions. Appl. Math. Lett. 84, 111-117 (2018)

18. Nieto, J.J.: An abstract monotone iterative technique. Nonlinear Anal. 28, 1923-1933 (1997)

19. Ahmad, B., Sivasundaram, S.: Existence results for nonlinear impulsive hybrid boundary value problems involving fractional differential equations. Nonlinear Anal. Hybrid Syst. 3, 251-258 (2009)

20. Abbas, S., Benchohra, M.: Upper and lower solutions method for impulsive partial hyperbolic differential equations with fractional order. Nonlinear Anal. Hybrid Syst. 4, 406-413 (2010)

21. Wang, G., Ahmad, B., Zhang, L.: Impulsive anti-periodic boundary value problem for nonlinear differential equations of fractional order. Nonlinear Anal. 74, 792-804 (2011)

22. Wang, J., Feckan, M., Zhou, Y.: A survey on impulsive fractional differential equations. Fract. Calc. Appl. Anal. 19, 806-831 (2016)

23. Yukunthorn, W., Ahmad, B., Ntouyas, S.K., Tariboon, J.: On Caputo-Hadamard type fractional impulsive hybrid systems with nonlinear fractional integral conditions. Nonlinear Anal. Hybrid Syst. 19, 77-92 (2016)

24. Wang, G., Ahmad, B., Zhang, L., Nieto, J.J.: Comments on the concept of existence of solution for impulsive fractional differential equations. Commun. Nonlinear Sci. Numer. Simul. 19, 401-403 (2014)

25. Liu, S., Wang, J., Zhou, Y.: Optimal control of noninstantaneous impulsive differential equations. J. Franklin Inst. 354, 7668-7698 (2017)

26. Zhang, L., Nieto, J.J., Wang, G.: Extremal solutions for a nonlinear impulsive differential equations with multi-orders fractional derivatives. J. Appl. Anal. Comput. 7, 814-823 (2017)

27. Harrat, A., Nieto, J.J., Debbouche, A.: Solvability and optimal controls of impulsive Hilfer fractional delay evolution inclusions with Clarke sub-differential. J. Comput. Appl. Math. 344, 725-737 (2018)

28. Benchohra, M., Nieto, J.J., Ouahab, A.: Impulsive differential inclusions via variational method. Georgian Math. J. 24, 313-323 (2017)

29. Agarwal, R.P., Hristova, S., O'Regan, D.: Iterative techniques for the initial value problem for Caputo fractional differential equations with non-instantaneous impulses. Appl. Math. Comput. 334, 407-421 (2018)

30. Katugampola, U.N.: New approach to a generalized fractional integral. Appl. Math. Comput. 218, 860-865 (2015)

31. Katugampola, U.N.: A new approach to generalized fractional derivatives. Bull. Math. Anal. Appl. 6, 1-15 (2014)

32. Jarad, F., Abdeljawad, T., Baleanu, D.: On the generalized fractional derivatives and their Caputo modification. J. Nonlinear Sci. Appl. 10, 2607-2619 (2017)

33. Krasnoselskii, M.A.: Two remarks on the method of successive approximations. Usp. Mat. Nauk 10, 123-127 (1955)

34. Heikkila, S., Lakshmikantham, V.: Monotone Iterative Techniques for Discontinuous Nonlinear Differential Equations. Marcel Dekker, New York (1994)

\section{Submit your manuscript to a SpringerOpen ${ }^{\circ}$ journal and benefit from:}

- Convenient online submission

- Rigorous peer review

- Open access: articles freely available online

- High visibility within the field

- Retaining the copyright to your article

Submit your next manuscript at $>$ springeropen.com 